\title{
Q-ADAPTED QUANTUM STOCHASTIC INTEGRALS AND DIFFERENTIALS IN FOCK SCALE
}

\author{
VIACHESLAV BELAVKIN and MATTHEW BROWN \\ School of Mathematical Sciences, University Park \\ Nottingham, NG7 2RD, UK \\ E-mail: viacheslav.belavkin@nottingham.ac.uk,pmxmb1@nottingham.ac.uk
}

\begin{abstract}
In this paper we first introduce the Fock-Guichardet formalism for the quantum stochastic (QS) integration, then the four fundamental processes of the dynamics are introduced in the canonical basis as the operator-valued measures, on a space-time $\sigma$-field $\mathfrak{F}_{\mathbb{X}}$, of the QS integration. Then rigorous analysis of the QS integrals is carried out, and continuity of the QS derivative $\mathbf{D}$ is proved. Finally, Q-adapted dynamics is discussed, including Bosonic $(\mathrm{Q}=\mathrm{I})$, Fermionic $(\mathrm{Q}=-\mathrm{I})$, and monotone $(\mathrm{Q}=\mathrm{O})$ quantum dynamics. These may be of particular interest to quantum field theory, quantum open systems, and quantum theory of stochastic processes.
\end{abstract}

1. Introduction. Non-commutative generalization of the Itô stochastic calculus, developed in [1, 2, 9, 15, 17, 18, gave an adequate mathematical tool for studying the behavior of open quantum dynamical systems singularly interacting with a boson quantumstochastic field. Quantum stochastic calculus also made it possible to solve an old problem of describing such systems with continuous observation and constructing a quantum filtration theory which would explain a continuous spontaneous collapse under the action of such observation [4, 5, 8. This gave examples of stochastic non-unitary, non-stationary, and even non-adapted evolution equations in a Hilbert space whose solution requires a proper definition of chronologically ordered quantum stochastic semigroups, and exponents of operators, by extending the notion of the multiple stochastic integral to noncommuting objects.

Here is the first part of an outline of the solution to this important problem by developing a $Q$-adapted form of the new quantum stochastic calculus constructed in [7] in a natural scale of Fock spaces. It is based on an explicit definition, introduced in [6],

2010 Mathematics Subject Classification: Primary 60H99; Secondary 60 G99.

Key words and phrases: Quantum stochastic calculus, non-adapted stochastic integrals, white noise analysis, quantum stochastic processes.

The paper is in final form and no version of it will be published elsewhere. 
of the non-adapted quantum stochastic integral, as a non-commutative generalization of the Skorokhod integral [19] represented in the Fock space. The point derivative of the quantum stochastic calculus is discussed as an operator on the scaled Fock space, and consequentially the single integral operator-kernels are derived from the operatorkernels of the multiple stochastic integral. These quantum stochastic derivatives are then presented in an explicit Q-adapted form, and we recover the Fermionic anti-commutation relation as well as the Bosonic commutator of the usual adapted process.

The approach used here is similar in spirit to the kernel calculus of Maassen-LindsayMeyer [15, 17, however the difference is that all the main objects are constructed not in terms of kernels but in terms of operators represented in the Fock space. In addition we employ a much more general notion of multiple stochastic integral, non-adapted in general but focusing now on Q-adapted processes, which reduces to the notion of the kernel representation of an operator only in the case of a scalar (non-random) operatorfunction under the integral. The possibility of defining a non-adapted single integral in terms of the kernel calculus was shown by Lindsay [14, but the notion of the multiple quantum-stochastic integral was introduced in [7].

2. Rigged Guichardet-Fock space. Let $(\mathbb{X}, \lambda)$ be an essentially ordered space, that is, a measurable space $\mathbb{X}$ with a $\sigma$-finite measure $\lambda: \mathfrak{F}_{\mathbb{X}} \ni \triangle \mapsto \lambda(\triangle) \geq 0$ and an ordering relation $x \leq x^{\prime}$ with the property that any $n$-tuple $\left(x_{1}, \ldots, x_{n}\right) \in \mathbb{X}^{n}$ can be identified up to a permutation with a chain $\varkappa=\left\{x_{1}<\ldots<x_{n}\right\}$ modulo the product measure $\prod_{i=1}^{n} \mathrm{~d} x_{i}$ of $\mathrm{d} x:=\lambda(\mathrm{d} x)$. In other words, we assume that the measurable ordering is almost total, that is, for any $n$ the product measure of $n$-tuples $s \in \mathbb{X}^{n}$ with components $\left(x_{1}, \ldots, x_{n}\right)$ that are not comparable is zero. Hence, in particular, it follows that the measure $\lambda$ on $\mathbb{X}$ is atomless and we may assume that this essentially total ordering on $\mathbb{X}$ is induced from the linear order in $\mathbb{R}_{+}$by a measurable map $t: \mathbb{X} \rightarrow \mathbb{R}_{+}$relatively to which $\lambda$ is absolutely continuous with respect to the Lebesgue measure $\mathrm{d} t$ on $\mathbb{R}_{+}$in the sense of admitting the disintegration

$$
\left\langle f \circ t, 1_{\triangle}\right\rangle_{\lambda}:=\int_{\triangle} f(t(x)) \lambda(\mathrm{d} x)=\int_{0}^{\infty} f(t) \lambda_{\triangle}(t) \mathrm{d} t \equiv\left\langle f, \lambda_{\triangle}\right\rangle .
$$

Here $1_{\triangle}$ is the indicator of any integrable subset $\triangle \subseteq \mathbb{X}$ and $f$ is any essentially bounded function $f: \mathbb{R}_{+} \rightarrow \mathbb{C}$ and $\triangle \mapsto \lambda_{\triangle}(t)$ is defined by duality as a positive measure on $\mathbb{X}$ for each $t \in \mathbb{R}_{+}$. In any case we will fix a map $t$ that the above condition holds and $t(x)<t\left(x^{\prime}\right)$ if $x<x^{\prime}$, interpreting $t(x)$ as the time at the point $x \in \mathbb{X}$. For example, $t(x)=t$ for $x=(\vec{x}, t)$ if $\mathbb{X}=\mathbb{R}^{d} \times \mathbb{R}_{+}$is the $(d+1)$-dimensional space-time with the casual ordering $[3$ and $\mathrm{d} x=\mathrm{d} \vec{x} \mathrm{~d} t$, where $\mathrm{d} \vec{x}$ is the standard volume element on $d$-dimensional space $\mathbb{R}^{d} \ni \vec{x}$.

We shall identify the finite chains $\varkappa$ with increasingly indexed $n$-tuples $\left(x_{1}, \ldots, x_{n}\right) \equiv s$ with $x_{i} \in \mathbb{X}, x_{1}<\ldots<x_{n}$, denoting by $\mathcal{X}=\sum_{n=0}^{\infty} \mathcal{X}_{n}$ the set of all finite chains as the union of the sets

$$
\mathcal{X}_{n}=\left\{s \in \mathbb{X}^{n}: x_{1}<\ldots<x_{n}\right\}
$$

with one-element $\mathcal{X}_{0}=\{\emptyset\}$ containing the empty chain as a subset of $\mathbb{X}: \emptyset=\mathbb{X}^{0}$. We introduce a measure 'element' $\mathrm{d} \varkappa=\prod_{x \in \varkappa} \mathrm{d} x$ on $\mathcal{X}$ induced by the direct sum 
$\bigoplus_{n=0}^{\infty} \lambda^{\otimes n}\left(\triangle_{n}\right), \triangle_{n} \in \mathfrak{F}_{\mathbb{X}}^{\otimes n}$ of product measures $\mathrm{d} \boldsymbol{s}=\prod_{i=1}^{n} \mathrm{~d} x_{i}$ on $\mathbb{X}^{n}$ with the unit mass $\mathrm{d} \varkappa=1$ at the only atomic point $\varkappa=\emptyset$.

Let $\left\{\mathfrak{k}_{x}: x \in \mathbb{X}\right\}$ be a family of Hilbert spaces $\mathfrak{k}_{x}$, let $\mathfrak{p}_{0}$ be an additive semigroup of nonnegative essentially measurable locally bounded functions $q: \mathbb{X} \rightarrow \mathbb{R}_{+}$with zero included $0 \in \mathfrak{p}_{0}$, and let $\mathfrak{p}_{1}=\left\{1+q_{0}: q_{0} \in \mathfrak{p}_{0}\right\}$. For example, in the case $\mathbb{X}=\mathbb{R}^{d} \times \mathbb{R}_{+}$ by $\mathfrak{p}_{1}$ we mean the set of polynomials $q(x)=1+\sum_{k=0}^{m} c_{k}|\vec{x}|^{k}$ with respect to the modulus $|\vec{x}|=\left(\Sigma x_{i}^{2}\right)^{1 / 2}$ of a vector $\vec{x} \in \mathbb{R}^{d}$ with coefficients $c_{k} \geq 0$. We denote by $K_{\star}(q)$ the Hilbert space of essentially measurable vector-functions $\mathrm{k}: x \mapsto \mathrm{k}(x) \in \mathfrak{k}_{x}$ which are square integrable with the weight $q \in \mathfrak{p}_{1}$ :

$$
\|\mathrm{k}\|(q)=\left(\int\|\mathrm{k}(x)\|_{x}^{2} q(x) \mathrm{d} x\right)^{1 / 2}<\infty .
$$

With $q \geq 1$, any space $K_{\star}(q)$ can be embedded into the Hilbert space $\mathfrak{k}=K_{\star}(1)$, and the intersection $\bigcap_{q \in \mathfrak{p}_{1}} K_{\star}(q) \subseteq \mathfrak{k}$ can be identified with the projective limit $K_{+}=$ $\lim _{q \rightarrow \infty} K_{\star}(q)$. This follows from the facts that the function $\|\mathrm{k}\|(q)$ is increasing: $q \leq p \Rightarrow\|\mathrm{k}\|(q) \leq\|\mathrm{k}\|(p)$, and so $K_{\star}(p) \subseteq K_{\star}(q)$, and that the set $\mathfrak{p}_{1}$ is directed in the sense that for any $q=1+r$ and $p=1+s, r, s \in \mathfrak{p}_{0}$, there is a function in $\mathfrak{p}_{1}$ majorizing $q$ and $p$ (we can take for example $q+p-1=1+r+s \in \mathfrak{p}_{1}$ ). In the case of polynomials $q \in \mathfrak{p}_{1}$ on $\mathbb{X}=\mathbb{R}^{d} \times \mathbb{R}_{+}$the decreasing family $\left\{K_{\star}(q)\right\}$, where $\mathfrak{k}_{x}=\mathbb{C}$, is identical with the integer Sobolev scale of vector fields $\mathrm{k}: \mathbb{R}^{d} \rightarrow L^{2}\left(\mathbb{R}_{+}\right)$with values $\mathrm{k}(x)(t)=\mathrm{k}(x, t)$ in the Hilbert space $L^{2}\left(\mathbb{R}_{+}\right)$of square integrable functions on $\mathbb{R}_{+}$. If we replace $\mathbb{R}^{d}$ by $\mathbb{Z}^{d}$ and if we restrict ourselves to the positive part of the integer lattice $\mathbb{Z}^{d}$, then we obtain the Schwartz space in the form of vector fields $\mathrm{k} \in K_{+}$.

The dual space $K_{\star}^{-}$to $K_{+}$is the space of generalized vector-functions $\mathrm{f}(x)$ defining the continuous functionals

$$
\langle\mathrm{f} \mid \mathrm{k}\rangle=\int\langle\mathrm{f}(x) \mid \mathrm{k}(x)\rangle \mathrm{d} x, \quad \mathrm{k} \in K_{+} .
$$

It is the inductive limit $K_{-}=\lim _{q \rightarrow 0} K_{\star}(q)$ in the opposite scale $\left\{K_{\star}(q): q \in \mathfrak{p}_{-}\right\}$, where $\mathfrak{p}_{-}$is the set of functions $q: \mathbb{X} \rightarrow(0,1]$ such that $1 / q \in \mathfrak{p}_{1}$, which is the union $\bigcup_{q \in \mathfrak{p}_{-}} K_{\star}(q)$ of the inductive family of Hilbert spaces $K_{\star}(q), q \in \mathfrak{p}_{-}$, with the norms $\|\mathrm{k}\|(q)$, containing as the minimal the space $\mathcal{K}_{*}=K_{\star}(1)$. Thus we obtain the Gel'fand chain

$$
K_{+} \subseteq K_{\star}\left(q_{+}\right) \subseteq \mathcal{K}_{*} \subseteq K_{\star}\left(q_{-}\right) \subseteq K_{-}
$$

in the extended scale $\left\{K_{\star}(q): q \in \mathfrak{p}\right\}$, where $\mathfrak{p}=\mathfrak{p}_{-} \cup \mathfrak{p}_{1}$, with $q_{+} \in \mathfrak{p}_{1}, q_{-} \in \mathfrak{p}_{-}$. The dual space $K_{+}^{\star}=K^{-}$is the space of the continuous linear functionals on $K_{+}$containing the Hilbert space $\mathcal{K}$ called the rigged space with respect to the dense subspace $K^{+}=K_{-}^{\star}$ of $\mathcal{K}$ equipped with the projective convergence in the scale $\left\|\mathrm{k}^{*}\right\|(q)=\|\mathrm{k}\|(q)$ for $q \in \mathfrak{p}_{1}$.

We can similarly define a Fock-Gel'fand triple $\left(F_{+}, \mathcal{F}_{*}, F_{-}\right)$with

$$
F_{+}=\bigcap_{q \in \mathfrak{p}_{1}} F_{\star}(q), \quad \mathcal{F}_{*}=F_{\star}(1), \quad F_{-}=\bigcup_{q \in \mathfrak{p}_{-}} F_{\star}(q),
$$

for the Hilbert scale $\left\{F_{\star}(q): q \in \mathfrak{p}\right\}$ of the symmetric Fock spaces $F_{\star}(q)=\bigoplus_{n=0}^{\infty} K_{\star}^{(n)}(q)$ over $K_{\star}(q)$, where $K_{\star}^{(0)}(q)=\mathbb{C}, K_{\star}^{(1)}(q)=K_{\star}(q)$, and each $K_{\star}^{(n)}(q)$ for $n>1$ is given by the product weight $q_{n}\left(x_{1}, \ldots, x_{n}\right)=\prod_{i=1}^{n} q\left(x_{i}\right)$ on $\mathbb{X}^{n}$. We shall consider the Guichardet [10] representation of the symmetric tensor-functions $\psi_{n} \in K_{\star}^{(n)}(q)$ regarding them as 
the restrictions $\psi \mid \mathcal{X}_{n}$ of the functions $\psi: \varkappa \mapsto \psi(\varkappa) \in K_{\star}^{\otimes}(\varkappa)$ with sections in the Hilbert products $K_{\star}^{\otimes}(\varkappa)=\bigotimes_{x \in \varkappa} \mathfrak{k}_{x}$, square integrable with the product weight $q(\varkappa)=$ $\prod_{x \in \varkappa} q(x)$ :

$$
\|\psi\|(q)=\left(\int\|\psi(\varkappa)\|^{2} q(\varkappa) \mathrm{d} \varkappa\right)^{1 / 2}<\infty .
$$

The integral here is over all chains $\varkappa \in \mathcal{X}$ and defines the pairing on $F_{+}$by

$$
\langle\psi \mid \psi\rangle=\int\langle\psi(\varkappa) \mid \psi(\varkappa)\rangle \mathrm{d} \varkappa, \quad \psi \in F_{+} .
$$

In more detail we can write this in the form

$$
\int\|\psi(\varkappa)\|^{2} q(\varkappa) \mathrm{d} \varkappa=\sum_{n=0}^{\infty} \int_{0 \leq t_{1}<\ldots<t_{n}<\infty}\left\|\psi\left(x_{1}, \ldots, x_{n}\right)\right\|^{2} \prod_{i=1}^{n} q\left(x_{i}\right) \mathrm{d} x_{i},
$$

where the $n$-fold integrals for $\psi_{n} \in K_{\star}^{(n)}$ are taken over simplex domains $\mathcal{X}_{n}=\left\{s \in \mathbb{X}^{n}\right.$ : $\left.t\left(x_{1}\right)<\ldots<t\left(x_{n}\right)\right\}$.

One can easily establish an isomorphism between the space $F_{\star}(q)$ and the symmetric (or antisymmetric) Fock space over $K_{\star}(q)$ with a nonatomic measure $\mathrm{d} x$ in $\mathbb{X}$. It is defined by the isometry

$$
\|\psi\|(q)=\left(\sum_{n=0}^{\infty} \frac{1}{n !} \int \cdots \int\left\|\psi\left(x_{1}, \ldots, x_{n}\right)\right\|^{2} \prod_{i=1}^{n} q\left(x_{i}\right) \mathrm{d} x_{i}\right)^{1 / 2},
$$

where the functions $\psi\left(x_{1}, \ldots, x_{n}\right)$ can be extended to the whole of $\mathbb{X}^{n}$ in a symmetric (or antisymmetric) way uniquely up to the measure zero due to nonatomicity of $\mathrm{d} x$ on $\mathbb{X}$.

3. Explicit definition of QS integrals. Let $\mathfrak{h}$ be a Hilbert space called the initial space for the Hilbert products $\mathcal{H}_{*}=\mathfrak{h} \otimes \mathcal{K}_{*}$ and $\mathcal{G}_{*}=\mathfrak{h} \otimes \mathcal{F}_{*}$. We consider the Hilbert scale $G_{\star}(q)=\mathfrak{h} \otimes F_{\star}(q), q \in \mathfrak{p}$, of complete tensor products of $\mathfrak{h}$ and the Fock spaces over $K_{\star}(q)$, and we put

$$
G_{+}=\bigcap G_{\star}(q), \quad G_{-}=\bigcup G_{\star}(q),
$$

which constitute the Gel'fand triple $G_{+} \subseteq \mathcal{G}_{*} \subseteq G_{-}$dual to $G^{+} \subseteq \mathcal{G} \subseteq G^{-}$of the Hermitian adjoint bra-spaces $G^{+}=G_{+}^{*}, \mathcal{G}=\mathcal{G}_{*}^{*}, G^{-}=G_{-}^{*}$.

Let $\left(\mathrm{D}_{\nu}^{\mu}\right)_{\nu=0,+}^{\mu=-,}$ be a quadruple of functions $\mathrm{D}_{\nu}^{\mu}$ on $\mathbb{X}$ with kernel values $\mathrm{D}_{\nu}^{\mu}(x): G_{+} \rightarrow$ $G_{-}$for $\mathfrak{k}_{x}=\mathbb{C}$, or, if $\mathfrak{k}_{x} \neq \mathbb{C}$, as continuous operators

$$
\begin{array}{ll}
\mathrm{D}_{+}^{-}(x): G_{+} \rightarrow G_{-}, & \mathrm{D}_{\circ}^{\circ}(x): \mathfrak{k}_{x} \otimes G_{+} \rightarrow \mathfrak{k}_{x} \otimes G_{-}, \\
\mathrm{D}_{+}^{\circ}(x): \mathfrak{k}_{x} \otimes G_{+} \rightarrow G_{-}, & \mathrm{D}_{\circ}^{-}(x): \mathfrak{k}_{x} \otimes G_{+} \rightarrow G_{-} .
\end{array}
$$

The continuity means that there is a $q \in \mathfrak{p}_{1}$ such that these operators are bounded from $G_{\star}(q) \supseteq G_{+}$to $G(q)^{\star} \subseteq G_{-}$, where $G(q)^{\star}=G_{\star}\left(q^{-1}\right)$. We assume that $\mathrm{D}_{+}^{-}(x)$ is locally integrable in the sense that

$$
\exists q \in \mathfrak{p}_{1}:\left\|\mathrm{D}_{+}^{-}\right\|_{q, t}^{(1)}=\int_{\mathbb{X}^{t}}\left\|\mathrm{D}_{+}^{-}(x)\right\|_{q} \mathrm{~d} x<\infty \quad \forall t<\infty,
$$

where $\mathbb{X}^{t}=\{x \in \mathbb{X}: t(x)<t\}$, and

$$
\|\mathrm{D}\|_{q}=\sup \left\{\|\mathrm{D} \chi\|\left(q^{-1}\right) /\|\chi\|(q)\right\}
$$

is the norm of the continuous operator $\mathrm{D}: G_{\star}(q) \rightarrow G_{\star}\left(q^{-1}\right)$ which defines a bounded Hermitian form $\mathrm{D}(\chi ; \chi):=\langle\chi \mid \mathrm{D} \chi\rangle$ on $G_{\star}(q)$. We also assume that $\mathrm{D}_{\circ}^{\circ}(x)$ is locally 
bounded with respect to a strictly positive function $s$ of $x$ such that $1 / s \in \mathfrak{p}_{0}$ in the sense that

$$
\exists q \in \mathfrak{p}_{1}:\left\|\mathrm{D}_{\circ}^{\circ}\right\|_{q, t}^{(\infty)}(s)=\underset{x \in \mathbb{X}^{t}}{\operatorname{ess} \sup }\left\{s(x)\left\|\mathrm{D}_{\circ}^{\circ}(x)\right\|_{q}\right\}<\infty \quad \forall t<\infty ;
$$

here $\|\mathrm{D}\|_{q}$ is the norm of the operator $\mathrm{D}: \mathfrak{k}_{x} \otimes G_{\star}(q) \rightarrow \mathfrak{k}_{x} \otimes G_{\star}\left(q^{-1}\right)$. Finally, we assume that $\mathrm{D}_{+}^{\circ}(x)$ and $\mathrm{D}_{\circ}^{-}(x)$ are locally square integrable with strictly positive function $r(x)$ such that $1 / r \in \mathfrak{p}_{0}$, in the sense that

$$
\exists q \in \mathfrak{p}_{1}:\left\|\mathrm{D}_{+}^{\circ}\right\|_{q, t}^{(2)}(r)<\infty, \quad\left\|\mathrm{D}_{\circ}^{-}\right\|_{q, t}^{(2)}(r)<\infty \quad \forall t<\infty,
$$

where $\|\mathrm{D}\|_{q, t}^{(2)}(r)=\left(\int_{\mathbb{X}^{t}}\|\mathrm{D}(x)\|_{q}^{2} r(x) \mathrm{d} x\right)^{1 / 2}$ and $\|\mathrm{D}\|_{q}$ are the respective norms of the operators

$$
\mathrm{D}_{+}^{\circ}(x): G_{\star}(q) \rightarrow \mathfrak{k}_{x} \otimes G_{\star}\left(q^{-1}\right), \quad \mathrm{D}_{\circ}^{-}(x): \mathfrak{k}_{x} \otimes G_{\star}(q) \rightarrow G_{\star}\left(q^{-1}\right) .
$$

Then for any $t \in \mathbb{R}_{+}$we can define a generalized quantum stochastic (QS) integral

$$
\boldsymbol{i}_{0}^{t}(\mathbf{D})=\int_{\mathbb{X}^{t}} \Lambda(\mathbf{D}, \mathrm{d} x), \quad \Lambda(\mathbf{D}, \triangle)=\sum_{\mu, \nu} \Lambda_{\nu}^{\mu}\left(\mathrm{D}_{\nu}^{\mu}, \triangle\right)
$$

introduced in [18] as the sum of four continuous operators $\Lambda_{\mu}^{\nu}\left(\mathrm{D}_{\nu}^{\mu}\right): G_{+} \rightarrow G_{-}$described as operator-measures on $\mathfrak{F}_{\mathbb{X}} \ni \triangle$ for $\triangle=\mathbb{X}^{t}$ with values

$$
\begin{aligned}
& {\left[\Lambda_{-}^{+}\left(\mathrm{D}_{+}^{-}, \triangle\right) \chi\right](\vartheta)=\int_{\triangle}\left[\mathrm{D}_{+}^{-}(x) \chi\right](\vartheta) \mathrm{d} x \quad \text { (preservation), }} \\
& {\left[\Lambda_{\circ}^{+}\left(\mathrm{D}_{+}^{\circ}, \triangle\right) \chi\right](\vartheta)=\sum_{x \in \triangle \cap \vartheta}\left[\mathrm{D}_{+}^{\circ}(x) \chi\right](\vartheta \backslash x) \quad \text { (creation) },} \\
& {\left[\Lambda_{-}^{\circ}\left(\mathrm{D}_{\circ}^{-}, \triangle\right) \chi\right](\vartheta)=\int_{\triangle}\left[\mathrm{D}_{\circ}^{-}(x) \dot{\chi}(x)\right](\vartheta) \mathrm{d} x \quad \text { (annihilation), }} \\
& {\left[\Lambda_{\circ}^{\circ}\left(\mathrm{D}_{\circ}^{\circ}, \triangle\right) \chi\right](\vartheta)=\sum_{x \in \triangle \cap \vartheta}\left[\mathrm{D}_{\circ}^{\circ}(x) \stackrel{\circ}{\chi}(x)\right](\vartheta \backslash x) \quad \text { (exchange). }}
\end{aligned}
$$

Here $\chi \in G_{+}, \vartheta \backslash x=\left\{x^{\prime} \in \vartheta: x^{\prime} \neq x\right\}$ denotes the chain $\vartheta \in \mathcal{X}$ from which the point $x \in \vartheta$ has been eliminated, and $\stackrel{\circ}{\chi}(x) \in \mathfrak{k}_{x} \otimes G_{+}$is the single point split $\stackrel{\circ}{\chi}(x)=\nabla_{x} \chi$, or point derivative, defined for each $\chi \in G_{+}$almost everywhere (namely, for $\vartheta \in \mathcal{X}: x \notin \vartheta$ ) as the function

$$
\left[\nabla_{x} \chi\right](\vartheta)=\chi(\vartheta \sqcup x) \equiv \stackrel{\circ}{\chi}(x, \vartheta),
$$

where the operation $\varkappa \sqcup x$ denotes the disjoint union $\vartheta=\varkappa \cup x, \varkappa \cap x=\emptyset$ of chains $\varkappa \in \mathcal{X}$ and $x \in \mathbb{X} \backslash \varkappa$ with pairwise comparable elements. Note that the point splitter $\nabla$ represents the Malliavin derivative [16] densely defined in Fock-Guichardet space as the bosonic annihilation operator $b(x): G_{+} \rightarrow \mathfrak{k}_{x} \otimes G_{+}$by $[b(x) \chi](\vartheta)=\stackrel{\circ}{\chi}(x, \vartheta)$ where one can take $\dot{\chi}(x, \vartheta)=0$ if $x \in \vartheta$, and its right inverse operator $\left[\nabla_{x}^{*} \psi\right](\vartheta)=\psi(x, \vartheta \backslash x)$ with $\left[\nabla_{x}^{*} \psi\right](\vartheta)=0$ if $x \notin \vartheta$ defines in this representation the Skorokhod non-adapted integral as the creation point integral

$$
\left[\nabla^{*} \psi\right](\vartheta)=\sum_{x \in \vartheta} \psi(x, \vartheta \backslash x)
$$

for any $\psi \in K_{-} \otimes G_{-}$. The continuity of this derivative as the projective limit map $G_{+} \rightarrow K_{+} \otimes G_{+}$and the point integral as the adjoint map $K_{-} \otimes G_{-} \rightarrow G_{-}$will simply 
follow from the isometricity of the multiple point splitter and co-isometricity of the adjoint multiple point integral as defined below, originally established in [7].

4. Split operator and its properties. As it is proved below, we can consider the multiple bosonic annihilation operators $b^{\otimes}(v): \chi \mapsto \grave{\chi}(v)$ eliminating several points $v$ in $\mathcal{X}_{n}$, with $\left[b^{\otimes}(v) \chi\right](\vartheta)=0$ if $v \subseteq \vartheta$, as partial isometries on the projective limit $G_{+}$ into $G_{+}^{(n)}=K_{+}^{\otimes n} \otimes G_{+}$. They are described for each $v=\left\{x_{1}, \ldots, x_{n}\right\}$ in terms of the $n$-point split

$$
\left[\Delta_{v}^{(n)} \chi\right](\vartheta):=\chi(\vartheta \sqcup v) \equiv \chi^{(n)}(v, \vartheta), \quad v \in \mathcal{X}_{n},
$$

where we put $\dot{\chi}(v)=\chi^{(n)}(v)$ for $n=|v|$. It is defined almost everywhere $(\vartheta \cap v=\emptyset)$ on $\vartheta \in \mathcal{X}$ as the $n$-th order $\Delta_{v_{n}}=\bigotimes_{x \in v_{n}} \nabla_{x}$ point (or Malliavin) derivative [16] such that $\Delta^{(0)}=\mathrm{I}$ and $\Delta^{(1)}=\nabla$. These $n$-tuple annihilations, densely defined as operators from $G_{+}$into $\mathfrak{k}^{\otimes}(v) \otimes G_{+}$, are not continuous for each $v \in \mathcal{X}_{n}$ (except $v=\emptyset$ corresponding to $n=0$ for which $\left.b^{\otimes}(\emptyset)=\mathrm{I}\right)$, but they define projective-continuous linear maps into the space $G_{+}^{(n)}$ of functions $v \mapsto \phi(v)$ on $\mathcal{X}_{n} \subset \mathcal{X}$ for each $n \in \mathbb{N}$, and therefore have the adjoints $G_{+}^{(n)} \rightarrow G_{+}$. This follows from the projective contractivity of the maps $\Delta^{(n)}$ : $G_{+} \rightarrow G_{+}^{(n)}$, and their adjoints, such that each function $\chi^{(n)}=\Delta^{(n)} \chi$ is square-integrable on $\mathcal{X}_{n}$ with any $q_{0} \in \mathfrak{p}_{0}$ being a component of the isometric operator

$$
\Delta \chi=\int_{\mathcal{X}}^{\oplus} \Delta_{v} \chi \mathrm{d} v=\bigoplus_{n=0}^{\infty} \Delta^{(n)} \chi \equiv \dot{\chi} .
$$

The projective isometricity of the linear operator $\Delta=\int_{\mathcal{X}}^{\oplus} \Delta_{v} \mathrm{~d} v$, called the multiple point splitter,

$$
\Delta: G_{\star}\left(q_{0}+q_{1}\right) \rightarrow F_{\star}\left(q_{0}\right) \otimes G_{\star}\left(q_{1}\right)
$$

is established in the following lemma.

Lemma 4.1. The linear map $\Delta: \chi \mapsto\left[\Delta^{(n)} \chi\right]$ defined as $\Delta \chi=\bigoplus_{n=0}^{\infty} \Delta^{(n)} \chi$ in (4) for all $v \in \mathcal{X}$ is a projective isometry on Hilbert scale $\left\{G_{\star}(q): q \in \mathfrak{p}\right\}$ into the scale $\left\{F_{\star}\left(q_{0}\right) \otimes G_{\star}\left(q_{1}\right): q_{0} \in \mathfrak{p}_{0}, q_{1} \in \mathfrak{p}_{1}\right\}$ such that

$$
\|\Delta \chi\|\left(q_{0}, q_{1}\right)=\|\chi\|\left(q_{0}+q_{1}\right) .
$$

The adjoint co-isometric operator $\left\langle\Delta^{*} \psi \mid \chi\right\rangle=\langle\psi \mid \Delta \chi\rangle$, defined on $\psi \in F_{\star}\left(q_{0}\right) \otimes G_{\star}\left(q_{1}\right)$ as the multiple point integral $\Delta^{*}=\sum \Delta_{n}^{*}$, is a contraction from $F_{\star}\left(q_{0}^{-1}\right) \otimes G_{\star}\left(q_{1}^{-1}\right)$ into any $G_{\star}\left(q^{-1}\right)$ with $q \geq q_{0}+q_{1}$ such that $\left(\Delta_{n}^{*}\right)^{*}=\Delta^{(n)}$. In particular,

$$
\left[\Delta_{n}^{*} \psi\right](\vartheta)=\sum_{\mathcal{X}_{n} \ni v \subseteq \vartheta} \psi(v, \vartheta \backslash v), \quad \vartheta \in \mathcal{X}
$$

defines for $\psi(v, \varkappa)=\psi_{n}(v) \otimes \chi(\varkappa)$ the $n$-th order Skorokhod integral

$$
\left[S_{n}\left(\psi_{n}\right) \chi\right](\vartheta)=\sum_{\mathcal{X}_{n} \ni v \subseteq \vartheta} \psi_{n}(v) \otimes \chi(\vartheta \backslash v)=\left[\Delta_{n}^{*}\left(\psi_{n} \otimes \chi\right)\right](\vartheta)
$$

of $\psi_{n} \in K_{\star}^{(n)}\left(q_{0}^{-1}\right)$ on $\chi \in G_{\star}\left(q_{1}^{-1}\right)$.

Proof. We first of all establish the principal formula of the multiple integration

$$
\int \sum_{v \subseteq \vartheta} f(v, \vartheta \backslash v) \mathrm{d} \vartheta=\iint f(v, \varkappa) \mathrm{d} v \mathrm{~d} \varkappa \quad \forall f \in L^{1}(\mathcal{X} \times \mathcal{X}),
$$


which will allow us to define the adjoint operator $\Delta^{*}$. Let $f(v, \varkappa)=g(v) h(\varkappa)$ be the product of integrable complex functions on $\mathcal{X}$ of the form $g(v)=\prod_{x \in v} g(x), h(\varkappa)=$ $\prod_{x \in \varkappa} h(x)$ for any $v, \varkappa \in \mathcal{X}$. Employing the binomial formula

$$
\sum_{v \subseteq \vartheta} g(v) h(\vartheta \backslash v)=\sum_{v \sqcup \varkappa=\vartheta} \prod_{x \in v} g(x) \prod_{x \in \varkappa} h(x)=\prod_{x \in \vartheta}(g(x)+h(x)),
$$

and also the equality $\int f(v) \mathrm{d} v=\exp \left\{\int f(x) \mathrm{d} x\right\}$ for $f(v)=\prod_{x \in v} f(x)$, we obtain the formula

$$
\int \sum_{v \subseteq \vartheta} g(v) h(\vartheta \backslash v) \mathrm{d} \vartheta=\exp \left\{\int(g(x)+h(x)) \mathrm{d} x\right\}=\iint g(v) h(\varkappa) \mathrm{d} v \mathrm{~d} \varkappa,
$$

which proves 6 on a set of product functions $f$ dense in $L^{1}(\mathcal{X} \times \mathcal{X})$.

Applying this formula to the scalar product $\langle\psi(v, \varkappa) \mid \psi(v, \varkappa)\rangle \in L^{1}(\mathcal{X} \times \mathcal{X})$, we obtain

$$
\int \sum_{v \subseteq \vartheta}\langle\psi(v, \vartheta \backslash v) \mid \chi(\vartheta)\rangle \mathrm{d} \vartheta=\iint\langle\psi(v, \varkappa) \mid \chi(v \sqcup \varkappa)\rangle \mathrm{d} v \mathrm{~d} \varkappa,
$$

that is, $\left\langle\Delta^{*} \psi \mid \chi\right\rangle=\langle\psi \mid \Delta \chi\rangle$, where $[\Delta \chi](v, \varkappa)=\chi(\varkappa \sqcup v) \equiv \dot{\chi}(v, \varkappa)$. Choosing arbitrary $\psi \in F_{\star}\left(q_{0}^{-1}\right) \otimes G_{\star}\left(q_{1}^{-1}\right)$, we find that the annihilation operators $b(v) \chi=\left[\Delta_{v} \chi\right]$ define the isometry $\Delta: G_{\star}\left(q_{0}+q_{1}\right) \rightarrow F_{\star}\left(q_{0}\right) \otimes G_{\star}\left(q_{1}\right)$ with the operator $\Delta^{*}$ defined as co-isometry $F_{\star}\left(q_{0}^{-1}\right) \otimes G_{\star}\left(q_{1}^{-1}\right) \rightarrow G_{\star}\left(q^{-1}\right)$ for $q=q_{0}+q_{1}$ with respect to the standard pairing of dual spaces $G_{\star}(q)$ and $G_{\star}\left(q^{-1}\right)$ :

$$
\begin{aligned}
& \|\dot{\chi}\|^{2}\left(q_{0}, q_{1}\right)=\iint\|\dot{\chi}(v, \varkappa)\|^{2} q_{0}(v) q_{1}(\varkappa) \mathrm{d} v \mathrm{~d} \varkappa \\
& =\int \sum_{v \subseteq \vartheta}\|\chi(\vartheta)\|^{2} q_{0}(v) q_{1}(\vartheta \backslash v) \mathrm{d} \vartheta=\int\|\chi(\vartheta)\|^{2} \sum_{v \sqcup \varkappa=\vartheta} q_{0}(v) q_{1}(\varkappa) \mathrm{d} \vartheta \\
& =\int\|\chi(\vartheta)\|^{2}\left(q_{0}+q_{1}\right)(\vartheta) \mathrm{d} \vartheta \equiv\|\chi\|^{2}\left(q_{0}+q_{1}\right) \leq\|\chi\|^{2}(q) \quad \forall q \geq q_{0}+q_{1} .
\end{aligned}
$$

Hence it follows that $\Delta$ is projective continuous operator from $G_{+}$to $F_{+} \otimes G_{+}$, where $F_{+}=\bigcap_{q \in \mathfrak{p}_{0}} F_{\star}(q)$, and in particular so is the one-point split $\chi(x, \varkappa)=\chi(x \sqcup \varkappa) \equiv \grave{\chi}(x, \varkappa)$ from $G_{+}$to $K_{+} \otimes G_{+}$, as a contracting map $G_{\star}\left(q_{0}+q_{1}\right) \rightarrow F_{\star}\left(q_{0}\right) \otimes G_{\star}\left(q_{1}\right)$ for all $q_{0} \in \mathfrak{p}_{0}$, $q_{1} \in \mathfrak{p}$. The lemma is proved.

REMARK. Because the explicit form of both the creation and annihilation operators' norms may not be obvious we shall review them here for the reader's familiarization.

$$
\|\Delta\|_{q_{0}+q_{1}}=\sup _{\chi} \frac{\|\Delta \chi\|\left(q_{0}, q_{1}\right)}{\|\chi\|\left(q_{0}+q_{1}\right)}=\sup _{\chi, \psi} \frac{|\langle\psi \mid \Delta \chi\rangle|}{\|\psi\|\left(q_{0}^{-1}, q_{1}^{-1}\right)\|\chi\|\left(q_{0}+q_{1}\right)}
$$

and

$$
\left\|\Delta^{*}\right\|^{q_{0}, q_{1}}=\sup _{\psi} \frac{\left\|\Delta^{*} \psi\right\|\left(\left(q_{0}+q_{1}\right)^{-1}\right)}{\|\psi\|\left(q_{0}^{-1}, q_{1}^{-1}\right)}=\sup _{\chi, \psi} \frac{\left|\left\langle\Delta^{*} \psi \mid \chi\right\rangle\right|}{\|\psi\|\left(q_{0}^{-1}, q_{1}^{-1}\right)\|\chi\|\left(q_{0}+q_{1}\right)}
$$

where $\psi \in F_{\star}\left(q_{0}^{-1}\right) \otimes G_{\star}\left(q_{1}^{-1}\right)$ and $\chi \in G_{\star}\left(q_{0}+q_{1}\right)$, indeed $\Delta^{*}: F_{\star}\left(q_{0}^{-1}\right) \otimes G_{\star}\left(q_{1}^{-1}\right) \rightarrow$ $G_{\star}\left(\left(q_{0}+q_{1}\right)^{-1}\right)$ and $\Delta: G_{\star}\left(q_{0}+q_{1}\right) \rightarrow F_{\star}\left(q_{0}\right) \otimes G_{\star}\left(q_{1}\right)$. By virtue of the fact that $\langle\psi \mid \Delta \chi\rangle=\left\langle\Delta^{*} \psi \mid \chi\right\rangle$ these two norms are equal, and they are equal to 1 . 
5. Multiple QS integrals and their continuity. We are now ready to prove the inductive continuity of the integral (2) with respect to $\mathbf{D}=\left[\mathrm{D}_{\nu}^{\mu}\right]$ by showing the inequality

$$
\left\|\left(i_{0}^{t}(\mathbf{D}) \chi\right)\right\|\left(p^{-1}\right) \leq\|\mathrm{D}\|_{q, t}^{s}(r)\|\chi\|(p) \quad \forall p \geq r^{-1}+q+s^{-1},
$$

where $\|\mathrm{D}\|_{q, t}^{s}(r)=\left\|\mathrm{D}_{+}^{-}\right\|_{q, t}^{(1)}+\left\|\mathrm{D}_{+}^{\circ}\right\|_{q, t}^{(2)}(r)+\left\|\mathrm{D}_{\circ}^{-}\right\|_{q, t}^{(2)}(r)+\left\|\mathrm{D}_{\circ}^{\circ}\right\|_{q, t}^{(\infty)}(s)$. We will establish this inequality as the single-integral case of the corresponding inequality for the generalized multiple QS integral 7

$$
\left[\boldsymbol{r}_{0}^{t}(\mathrm{M}) \chi\right](\vartheta)=\sum_{v_{\circ}^{\circ} \sqcup v_{+}^{\circ} \subseteq \vartheta^{t}} \int_{\mathcal{X}^{t}} \int_{\mathcal{X}^{t}}\left[\mathrm{M}(\boldsymbol{v}) \stackrel{\circ}{\chi}\left(v_{\circ}^{-} \sqcup v_{\circ}^{\circ}\right)\right]\left(v_{-}^{\circ}\right) \mathrm{d} v_{+}^{-} \mathrm{d} v_{\circ}^{-}
$$

where $\vartheta^{t}=\vartheta \cap \mathbb{X}^{t}, \mathcal{X}^{t}=\left\{\vartheta \in \mathcal{X}: \vartheta \subset \mathbb{X}^{t}\right\}$ and the sum is taken over all decompositions $\vartheta=v_{-}^{\circ} \sqcup v_{\circ}^{\circ} \sqcup v_{+}^{\circ}$ such that $v_{\circ}^{\circ} \in \mathcal{X}^{t}$ and $v_{+}^{\circ} \in \mathcal{X}^{t}$. The multi-integrand $\mathrm{M}(\boldsymbol{v})$ defines the values

$$
\mathrm{M}\left(v_{.}\right)=\delta_{\emptyset}\left(v_{\cdot}^{+}\right) \mathrm{M}(\boldsymbol{v}) \delta_{\emptyset}\left(v_{-}^{\cdot}\right), \quad \boldsymbol{v}=\left(\begin{array}{cc}
v_{+}^{-} & v_{\circ}^{-} \\
v_{+}^{\circ} & v_{\circ}^{\circ}
\end{array}\right)
$$

of matrix elements $\mathrm{M}:(v)=\mathrm{M}\left(v^{*}\right), v=\bigsqcup_{\mu, \nu} v_{\nu}^{\mu}$, for a decomposable triangular tensoroperator $\mathbf{M} \equiv \mathbf{M}$ : defined on matrices $v_{:}=\left[v_{\nu}^{\mu}\right]_{\nu=-, o,+}^{\mu=-,+}$ whose elements are finite disjoint chains $v_{\nu}^{\mu}$, with $v_{\nu}^{\mu}=\emptyset$ for $\mu>\nu$ and with $\mathrm{M}\left(v_{.}^{*}\right)=0$ if $v^{+} \neq \emptyset$ or $v_{-}^{*} \neq \emptyset$. The other values of $\mathrm{M}\left(v^{*}\right)$ are defined by a kernel-operator function $\mathrm{M}(\boldsymbol{v})$ of the quadruple $\boldsymbol{v}=\left(v_{\nu}^{\mu}\right)_{\nu=+, \circ}^{\mu=-\circ}$ with continuous operator values $\mathrm{M}(\boldsymbol{v}): G_{+} \rightarrow G_{-}$in the scalar case $\mathfrak{k}_{x}=\mathbb{C}$. In the general case it is defined almost everywhere by its values on the chains $v_{\nu}^{\mu} \in \mathcal{X}$ in the continuous operators

$$
\mathrm{M}\left(\begin{array}{cc}
v_{+}^{-} & v_{\circ}^{-} \\
v_{+}^{\circ} & v_{\circ}^{\circ}
\end{array}\right): \mathfrak{k}_{v_{\circ}^{-}}^{\otimes} \otimes \mathfrak{k}_{v_{\circ}^{\circ}}^{\otimes} \otimes G_{+} \rightarrow \mathfrak{k}_{v_{\circ}^{\circ}}^{\otimes} \otimes \mathfrak{k}_{v_{+}^{\circ}}^{\otimes} \otimes G_{-} .
$$

We will assume that these operators are bounded from $G_{\star}(q)$ to $G_{\star}\left(q^{-1}\right)$ for some $q \in \mathfrak{p}_{1}$, such that $\|\mathrm{I}\|=1$, and that there exist strictly positive functions $r>0, r^{-1} \in \mathfrak{p}_{0}$, and $s>0, s^{-1} \in \mathfrak{p}_{0}$ such that

$$
\|\mathrm{M}\|_{q, t}^{s}(r)=\int_{\mathcal{X}^{t}}\left\|\mathrm{M}_{+}^{-}(v)\right\|_{q, t}^{s}(r) \mathrm{d} v<\infty \quad \forall t<\infty,
$$

where

$$
\left\|\mathrm{M}_{+}^{-}\left(v_{+}^{-}\right)\right\|_{q, t}^{s}(r)=\left(\int_{\mathcal{X}^{t}} \int_{\mathcal{X}^{t}} \operatorname{ess~sup}_{v_{\circ}^{\circ} \in \mathcal{X}^{t}}\left(s\left(v_{\circ}^{\circ}\right)\|\mathrm{M}(\boldsymbol{v})\|_{q}\right)^{2} r\left(v_{+}^{\circ} \sqcup v_{\circ}^{-}\right) \mathrm{d} v_{+}^{\circ} \mathrm{d} v_{\circ}^{-}\right)^{1 / 2},
$$

and $s(v)=\prod_{x \in v} s(x), r(v)=\prod_{x \in v} r(x)$.

We mention that the single integral (2) corresponds to the case

$$
\mathrm{M}\left(v^{\cdot}\right)=0 \quad \forall v^{\cdot}: \sum_{\mu \neq+, \nu \neq-}\left|v_{\nu}^{\mu}\right| \neq 1
$$

and $\mathrm{M}\left(\mathbf{x}_{\nu}^{\mu}\right)=\mathrm{D}_{\nu}^{\mu}(x)$ otherwise, where $\mathbf{x}_{\nu}^{\mu}$ denotes one of six 'atomic' triangular matrices $v:(\mathbf{x})=\left[v_{\lambda}^{\kappa}(\mathbf{x})\right]_{\lambda=-, \circ,+}^{\kappa=-, o,+} \equiv \mathbf{x}$ having all matrix elements $v_{\lambda}^{\kappa}(\mathbf{x})$ empty if $\mathbf{x} \neq \mathbf{x}_{\lambda}^{\kappa}$, but $v_{\nu}^{\mu}(\mathbf{x})=x$ for $\mathbf{x}=\mathbf{x}_{\nu}^{\mu}$. Note that integrand $\mathrm{M}\left(\mathbf{x}_{\nu}^{\mu}\right)$ is zero on the atomic matrices $\mathbf{x}_{\nu}^{+}$ and $\mathbf{x}_{-}^{\mu}$, otherwise $\mathrm{M}(\mathbf{x})=\mathrm{M}(\boldsymbol{x})$, given by the single-point kernel $\mathrm{M}(\boldsymbol{x})$ as a function of one of the four single-point tables $\boldsymbol{v}(x)=\left(v_{\lambda}^{\kappa}(\mathbf{x})\right)_{\lambda=+, \circ}^{\kappa=-, \circ} \equiv \boldsymbol{x}$ :

$$
\boldsymbol{x}_{+}^{-}=\left(\begin{array}{cc}
x, & \emptyset \\
\emptyset & \emptyset
\end{array}\right), \quad \boldsymbol{x}_{+}^{\circ}=\left(\begin{array}{cc}
\emptyset, & \emptyset \\
x & \emptyset
\end{array}\right), \quad \boldsymbol{x}_{\circ}^{-}=\left(\begin{array}{cc}
\emptyset, & x \\
\emptyset & \emptyset
\end{array}\right), \quad \boldsymbol{x}_{\circ}^{\circ}=\left(\begin{array}{ll}
\emptyset, & \emptyset \\
\emptyset & x
\end{array}\right),
$$


determined by an $x \in \mathbb{X}$. It follows from the next theorem that the function $\mathrm{M}(\boldsymbol{v})$ in (7) can be defined up to equivalence, whose kernel $\{\mathrm{M} \approx 0\}$ consists of all multiple integrands with $\|\mathrm{M}\|_{q, t}^{s}(r)=0$ for all $t \in \mathbb{R}_{+}$and for some $q, r, s$. In particular, $\mathrm{M}$ can be defined almost everywhere only for the tables $\boldsymbol{v}=\left(v_{\nu}^{\mu}\right)$ that give disjoint decompositions $v=$ $\bigsqcup_{\mu, \nu} v_{\nu}^{\mu}$ of the chains $v \in \mathcal{X}$, that is, it may have nonzero values only on $\boldsymbol{v}$ representable in the form $\boldsymbol{v}=\bigsqcup_{x \in v} \boldsymbol{x}$, where $\boldsymbol{x}$ is one of the atomic tables given by (9) with indices $\mu, \nu$ for $x \in v_{\nu}^{\mu}$.

THEOREM 5.1. Suppose that $\mathrm{M}(\boldsymbol{v})$ is a locally integrable function in the sense of (8) for some $q, r, s>0$. Then its integral (7) is a continuous operator $\mathrm{T}_{t}=\boldsymbol{\imath}_{0}^{t}(\mathrm{M})$ from $G_{+}$ to $G_{-}$satisfying the estimate

$$
\left\|\mathrm{T}_{t}\right\|_{p}=\sup _{\chi \in G_{\star}(p)}\left\{\left\|\mathrm{T}_{t} \chi\right\|\left(p^{-1}\right) /\|\chi\|(p)\right\} \leq\|\mathrm{M}\|_{q, t}^{s}(r)
$$

for any $p \geq r^{-1}+q+s^{-1}$. The operator $\mathrm{T}_{t}^{*}$, formally adjoint to $\mathrm{T}_{t}$ in $\mathcal{G}_{*}$, is the integral

$$
\boldsymbol{\imath}_{0}^{t}(\mathrm{M})^{*}=\boldsymbol{\imath}_{0}^{t}\left(\mathrm{M}^{\ddagger}\right), \quad \mathrm{M}^{\ddagger}\left(v_{.}^{*}\right)=\mathrm{M}\left(\tilde{v}_{:}\right)^{*}, \quad \widetilde{\left[v_{\nu}^{\mu}\right]}=\left[v_{-\mu}^{-\nu}\right]
$$

of $\delta_{\emptyset}\left(v^{+}\right) \mathrm{M}^{\star}(\boldsymbol{v}) \delta_{\emptyset}\left(v_{-}^{\cdot}\right)=\mathrm{M}^{\ddagger}\left(v_{.}^{*}\right)$, which is continuous from $G_{+}$to $G_{-}$, and satisfying $\left\|\mathrm{M}^{\star}\right\|_{q, t}^{s}(r)=\|\mathrm{M}\|_{q, t}^{s}(r)$ for $\mathrm{M}^{\star}(\boldsymbol{v})=\mathrm{M}\left(\boldsymbol{v}^{\prime}\right)^{*}$, where $\left(v_{\nu}^{\mu}\right)^{\prime}=\left(v_{-\mu}^{-\nu}\right)$. Moreover, the operator-valued function $t \mapsto \mathrm{T}_{t}$ has the quantum-stochastic differential $\mathrm{dT}_{t}=\mathrm{d} \boldsymbol{i}_{0}^{t}(\mathbf{D})$ in the sense that

$$
\boldsymbol{\imath}_{0}^{t}(\mathrm{M})=\mathrm{M}(\emptyset)+\boldsymbol{i}_{0}^{t}(\mathbf{D}), \quad \mathrm{D}_{\nu}^{\mu}(x)=\boldsymbol{\imath}_{0}^{t(x)}\left(\dot{\mathrm{M}}\left(\mathbf{x}_{\nu}^{\mu}\right)\right),
$$

defined by the quantum-stochastic derivatives $\mathbf{D}=\left[\mathrm{D}_{\nu}^{\mu}\right]$ with values (1) acting from $G_{\star}(p)$ to $G_{\star}\left(p^{-1}\right)$ and bounded almost everywhere:

$$
\left\|\mathrm{D}_{+}^{-}\right\|_{p, t}^{(1)} \leq\|\mathrm{M}\|_{q, t}^{s}(r), \quad\|\mathrm{D}\|_{p, t}^{(2)}(r) \leq\|\mathrm{M}\|_{q, t}^{s}(r), \quad\left\|\mathrm{D}_{\circ}^{\circ}\right\|_{p, t}^{(\infty)}(s) \leq\|\mathrm{M}\|_{q, t}^{s}(r)
$$

for $\mathrm{D}=\mathrm{D}_{\circ}^{-}$and $\mathrm{D}=\mathrm{D}_{+}^{\circ}, p \geq r^{-1}+q+s^{-1}$. This differential is defined in the form of the multiple integrals (7), with respect to $\boldsymbol{v}$, of the point derivatives $\dot{\mathrm{M}}\left(\mathbf{x}, v^{*}\right)=\mathrm{M}\left(v^{\cdot} \sqcup \mathbf{x}\right)$, where $\mathbf{x}$ is given by one of four atomic tables (9) at a fixed point $x \in \mathbb{X}$.

Proof. Using property 6 in the form

$$
\int \sum_{\sqcup v_{\nu}^{\circ}=\vartheta} f\left(v_{-}^{\circ}, v_{\circ}^{\circ}, v_{+}^{\circ}\right) \mathrm{d} \vartheta=\iiint f\left(v_{-}^{\circ}, v_{\circ}^{\circ}, v_{+}^{\circ}\right) \prod_{\nu} \mathrm{d} v_{\nu}^{\circ},
$$

it is easy to find that from the definition (7) for $\chi \in G_{+}$we have

$$
\begin{aligned}
& \int\left\langle\chi(\vartheta) \mid\left[\mathrm{T}_{t} \chi\right](\vartheta)\right\rangle \mathrm{d} \vartheta \\
& =\int_{\mathcal{X}^{t}} \mathrm{~d} v_{+}^{-} \int_{\mathcal{X}^{t}} \mathrm{~d} v_{+}^{\circ} \int_{\mathcal{X}^{t}} \mathrm{~d} v_{\circ}^{-} \int_{\mathcal{X}^{t}} \mathrm{~d} v_{\circ}^{\circ}\left\langle\stackrel{\chi}{\chi}\left(v_{\circ}^{\circ} \sqcup v_{+}^{\circ}\right) \mid \mathrm{M}(\boldsymbol{v}) \dot{\chi}\left(v_{\circ}^{-} \sqcup v_{\circ}^{\circ}\right)\right\rangle \\
& =\int_{\mathcal{X}^{t}} \mathrm{~d} v_{+}^{-} \int_{\mathcal{X}^{t}} \mathrm{~d} v_{+}^{\circ} \int_{\mathcal{X}^{t}} \mathrm{~d} v_{\circ}^{-} \int_{\mathcal{X}^{t}} \mathrm{~d} v_{\circ}^{\circ}\left\langle\mathrm{M}(\boldsymbol{v})^{*} \dot{\chi}\left(v_{\circ}^{\circ} \sqcup v_{+}^{\circ}\right) \mid \dot{\chi}\left(v_{\circ}^{-} \sqcup v_{\circ}^{\circ}\right)\right\rangle \\
& =\int\left\langle\left[\mathrm{T}_{t}^{*} \chi\right](\vartheta) \mid \chi(\vartheta)\right\rangle \mathrm{d} \vartheta,
\end{aligned}
$$

that is, $\mathrm{T}_{t}^{*}$ acts as $\boldsymbol{\imath}_{0}^{t}\left(\mathrm{M}^{\star}\right)$ in (7) with $\mathrm{M}^{\star}(\boldsymbol{v})=\mathrm{M}\left(\boldsymbol{v}^{\prime}\right)^{*}$, where $\left(v_{\nu}^{\mu}\right)^{\prime}=\left(v_{-\mu}^{-\nu}\right)$ with respect to the inversion $-:(-, \circ,+) \mapsto(+, \circ,-)$. More precisely, this yields $\left\|\boldsymbol{\imath}_{0}^{t}(\mathrm{M})\right\|_{p}=$ $\left\|\boldsymbol{\imath}_{0}^{t}\left(\mathrm{M}^{\star}\right)\right\|_{p}$, since $\|\mathrm{T}\|_{p}=\left\|\mathrm{T}^{*}\right\|_{p}$ by the definition 10 of $p$-norm and by

$$
\sup \{|\langle\chi \mid \mathrm{T} \chi\rangle| /\|\chi\|(p)\|\chi\|(p)\}=\sup \left\{\left|\left\langle\mathrm{T}^{*} \chi \mid \chi\right\rangle\right| /\|\chi\|(p)\|\chi\|(p)\right\} .
$$


We estimate the integral $\left\langle\chi \mid \mathrm{T}_{t} \chi\right\rangle$ using the Schwartz inequality

$$
\int\|\dot{\chi}(v)\|(q)\|\dot{\chi}(v)\|(q) s^{-1}(v) \mathrm{d} v \leq\|\dot{\chi}\|\left(s^{-1}, q\right)\|\dot{\chi}\|\left(s^{-1}, q\right)
$$

and the property $(6)$ of the multiple integral according to which

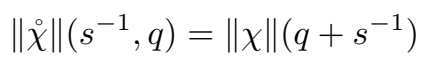

then

$$
\begin{aligned}
& \left|\left\langle\chi \mid \mathrm{T}_{t} \chi\right\rangle\right| \leq \int_{\mathcal{X}^{t}} \int_{\mathcal{X}^{t}} \int_{\mathcal{X}^{t}} \int_{\mathcal{X}^{t}}\left\|\stackrel{\circ}{\chi}\left(v_{\circ}^{\circ} \sqcup v_{+}^{\circ}\right)\right\|(q)\left\|\mathrm{M}(\boldsymbol{v}) \dot{\chi}\left(v_{\circ}^{\circ} \sqcup v_{\circ}^{-}\right)\right\|\left(q^{-1}\right) \mathrm{d}^{4} \boldsymbol{v} \\
& \quad \leq \int_{\mathcal{X}^{t}} \mathrm{~d} v_{\circ}^{\circ} \int_{\mathcal{X}^{t}} \int_{\mathcal{X}^{t}}\left\|\stackrel{\circ}{\chi}\left(v_{\circ}^{\circ} \sqcup v_{+}^{\circ}\right)\right\|(q)\left(\int_{\mathcal{X}^{t}}\|\mathrm{M}(\boldsymbol{v})\|_{q} \mathrm{~d} v_{+}^{-}\right)\left\|\stackrel{\chi}{\chi}\left(v_{\circ}^{-} \sqcup v_{\circ}^{\circ}\right)\right\|(q) \mathrm{d} v_{\circ}^{-} \mathrm{d} v_{+}^{\circ} \\
& \quad \leq \int_{\mathcal{X}^{t}} \mathrm{~d} v\left(\int_{\mathcal{X}^{t}}\left\|\grave{\chi}\left(v \sqcup v_{+}^{\circ}\right)\right\|^{2}(q) \frac{\mathrm{d} v_{+}^{\circ}}{r\left(v_{+}^{\circ}\right)} \int_{\mathcal{X}^{t}}\left\|\grave{\chi}\left(v \sqcup v_{\circ}^{-}\right)\right\|^{2}(q) \frac{\mathrm{d} v_{\circ}^{-}}{r\left(v_{\circ}^{-}\right)}\right)^{1 / 2}\left\|\mathrm{M}_{\circ}^{\circ}(v)\right\|_{q, t}(r) \\
& \quad=\int_{\mathcal{X}^{t}} \mathrm{~d} v\|\stackrel{\circ}{\chi}(v)\|\left(r^{-1}+q\right)\left\|\mathrm{M}_{\circ}^{\circ}(v)\right\|_{q, t}(r)\|\stackrel{\circ}{\chi}(v)\|\left(r^{-1}+q\right) \\
& \leq \underset{v \in \mathcal{X}^{t}}{\operatorname{ess~} \sup }\left\{s(v)\left\|\mathrm{M}_{\circ}^{\circ}(v)\right\|_{q, t}(r)\right\}\|\chi\|\left(r^{-1}+q+s^{-1}\right)\|\chi\|\left(r^{-1}+q+s^{-1}\right),
\end{aligned}
$$

where $\left\|\mathrm{M}_{\circ}^{\circ}\left(v_{\circ}^{\circ}\right)\right\|_{q, t}(r)=\left(\int_{\mathcal{X}^{t}} \int_{\mathcal{X}^{t}}\left(\int_{\mathcal{X}^{t}}\|\mathrm{M}(\boldsymbol{v})\|_{q} \mathrm{~d} v_{+}^{-}\right)^{2} r\left(v_{\circ}^{-} \sqcup v_{+}^{\circ}\right) \mathrm{d} v_{\circ}^{-} \mathrm{d} v_{+}^{\circ}\right)^{1 / 2}$, and $\mathrm{d}^{4} \boldsymbol{v}:=$ $\mathrm{d} v_{\circ}^{-} \mathrm{d} v_{\circ}^{\circ} \mathrm{d} v_{+}^{\circ} \mathrm{d} v_{+}^{-}$. Then since

$$
\underset{v \in \mathcal{X}^{t}}{\operatorname{ess} \sup }\left\{s(v)\left\|\mathrm{M}_{\circ}^{\circ}(v)\right\|_{q, t}(r)\right\} \leq\|\mathrm{M}\|_{q, t}^{s}(r),
$$

and since

$$
\sup _{\chi \in G_{\star}(p)} \frac{\|\mathrm{T} \chi\|\left(p^{-1}\right)}{\|\chi\|(p)}=\sup _{\chi \in G_{\star}(p)} \frac{|\langle\chi \mid \mathrm{T} \chi\rangle|}{\|\chi\|(p)\|\chi\|(p)},
$$

it follows that $\left\|\mathrm{T}_{t}\right\|_{p} \leq\|\mathrm{M}\|_{q, t}^{s}(r)$ for all $p \geq r^{-1}+q+s^{-1}$.

Now using the definition (7) and the property

$$
\int_{\mathcal{X}^{t}} \chi(\vartheta) \mathrm{d} \vartheta=\chi(\emptyset)+\int_{\mathbb{X}^{t}} \mathrm{~d} x \int_{\mathcal{X}^{t(x)}} \dot{\chi}(x, \vartheta) \mathrm{d} \vartheta,
$$

where $\stackrel{\circ}{\chi}(x, \vartheta)=\chi(\vartheta \sqcup x)$, it is easy to see that

$$
\begin{aligned}
& {\left[\left(\mathrm{T}_{t}-\mathrm{T}_{0}\right) \chi\right](\vartheta)=\left[\left(\boldsymbol{\imath}_{0}^{t}(\mathrm{M})-\mathrm{M}(\emptyset)\right) \chi\right](\vartheta)} \\
& \begin{array}{l}
\int_{\mathbb{X}^{t}} \mathrm{~d} x \sum_{v_{\circ}^{\circ} \sqcup v_{+}^{\circ} \subseteq \vartheta}^{t\left(v_{\nu}^{\circ}\right)<t(x)}\left\{\int _ { \mathcal { X } ^ { t ( x ) } } \mathrm { d } v _ { + } ^ { - } \left[\int _ { \mathcal { X } ^ { t ( s ) } } \mathrm { d } v _ { \circ } ^ { - } \left(\dot{\mathrm{M}}\left(\mathbf{x}_{+}^{-}, \boldsymbol{v}\right) \dot{\chi}\left(v_{\circ}^{-} \sqcup v_{\circ}^{\circ}\right)\right.\right.\right. \\
\left.\left.\left.+\dot{\mathrm{M}}\left(\mathbf{x}_{\circ}^{-}, \boldsymbol{v}\right) \dot{\chi}\left(x \sqcup v_{\circ}^{-} \sqcup v_{\circ}^{\circ}\right)\right)\right]\right\}\left(\vartheta \backslash v_{\circ}^{\circ} \backslash v_{+}^{\circ}\right)
\end{array} \\
& +\sum_{x \in \mathbb{X}^{t}} \int_{v_{\circ}^{\circ} \sqcup v_{+}^{\circ} \subseteq \vartheta}^{t\left(v_{\nu}^{\circ}\right)<t(x)}\left\{\int _ { \mathcal { X } ^ { t ( x ) } } \mathrm { d } v _ { + } ^ { - } \left[\int _ { \mathcal { X } ^ { t ( x ) } } \mathrm { d } v _ { \circ } ^ { - } \left(\dot{\mathrm{M}}\left(\mathbf{x}_{+}^{\circ}, \boldsymbol{v}\right) \dot{\chi}\left(v_{\circ}^{-} \sqcup v_{\circ}^{\circ}\right)\right.\right.\right. \\
& \left.\left.\left.\quad+\dot{\mathrm{M}}\left(\mathbf{x}_{\circ}^{\circ}, \boldsymbol{v}\right) \stackrel{\circ}{\chi}\left(x \sqcup v_{\circ}^{-} \sqcup v_{\circ}^{\circ}\right)\right)\right]\right\}\left(\vartheta \backslash v_{\circ}^{\circ} \backslash v_{+}^{\circ}\right) \\
& =\int_{\mathbb{X}^{t}} \mathrm{~d} x\left[\mathrm{D}_{+}^{-}(x) \chi+\mathrm{D}_{\circ}^{-}(x) \dot{\chi}(x)\right](\vartheta)+\sum_{x \in \mathbb{X}^{t}}\left[\mathrm{D}_{+}^{\circ}(x) \chi+\mathrm{D}_{\circ}^{\circ}(x) \grave{\chi}(x)\right](\vartheta \backslash x) .
\end{aligned}
$$


Consequently, $\mathrm{T}_{t}-\mathrm{T}_{0}=\sum \Lambda_{\mu}^{\nu}\left(\mathrm{D}_{\nu}^{\mu}, \mathbb{X}^{t}\right)$, where $\Lambda_{\nu}^{\mu}(\mathrm{D}, \triangle)$ are defined in (3) as operatorvalued measures on $\mathbb{X}$ of operator-functions

$$
\begin{aligned}
{\left[\mathrm{D}_{+}^{\mu}(x) \chi\right](\vartheta) } & =\sum_{v_{\circ}^{\circ} \sqcup v_{+}^{\circ} \subseteq \vartheta}^{t\left(v_{\nu}^{\circ}\right)<t(x)} \int_{\mathcal{X}^{t(x)}} \mathrm{d} v_{+}^{-} \int_{\mathcal{X}^{t(x)}} \mathrm{d} v_{\circ}^{-}\left[\dot{\mathrm{M}}\left(\mathbf{x}_{+}^{\mu}, \boldsymbol{v}\right) \stackrel{\chi}{\chi}\left(v_{\circ}^{-} \sqcup v_{\circ}^{\circ}\right)\right]\left(\vartheta_{-}^{\circ}\right), \\
{\left[\mathrm{D}_{\circ}^{\mu}(x) \dot{\chi}(x)\right](\vartheta) } & =\sum_{v_{\circ}^{\circ} \sqcup v_{+}^{\circ} \subseteq \vartheta}^{t\left(v_{\nu}^{\circ}\right)<t(x)} \int_{\mathcal{X}^{t(x)}} \mathrm{d} v_{+}^{-} \int_{\mathcal{X}^{t(x)}} \mathrm{d} v_{\circ}^{-}\left[\dot{\mathrm{M}}\left(\mathbf{x}_{\circ}^{\mu}, \boldsymbol{v}\right) \dot{\chi}\left(x \sqcup v_{\circ}^{-} \sqcup v_{\circ}^{\circ}\right)\right]\left(\vartheta_{-}^{\circ}\right),
\end{aligned}
$$

acting on $\chi \in G_{+}$and $\stackrel{\circ}{\chi}(v) \in \mathfrak{k}_{v}^{\otimes} \otimes G_{+}$, where $\vartheta_{-}^{\circ}=\vartheta \cap \overline{\left(v_{\circ}^{\circ} \sqcup v_{+}^{\circ}\right)}=\vartheta \backslash v_{\circ}^{\circ} \backslash v_{+}^{\circ}$. This can be written in terms of (7) as

$$
\mathrm{D}_{\nu}^{\mu}(x)=\boldsymbol{\imath}_{0}^{t}\left(\dot{\mathrm{M}}\left(\mathrm{x}_{\nu}^{\mu}\right)\right)
$$

Because of the inequality $\left\|\mathrm{T}_{t}\right\|_{p} \leq\|\mathrm{M}\|_{q, t}^{s}(r)$ for all $p \geq r^{-1}+q+s^{-1}$ we obtain $\left\|\mathrm{D}_{+}^{-}\right\|_{p, t}^{(1)} \leq$ $\|\mathrm{M}\|_{q, t}^{s}(r)$, since $\left\|\mathrm{D}_{+}^{-}(x)\right\|_{p} \leq\left\|\dot{\mathrm{M}}\left(\mathbf{x}_{+}^{-}\right)\right\|_{q, t(x)}^{s}(r)$ :

$$
\begin{aligned}
\int_{\mathbb{X}^{t}}\left\|\mathrm{D}_{+}^{-}(x)\right\|_{p} \mathrm{~d} x & \leq \int_{\mathbb{X}^{t}}\left\|\dot{\mathrm{M}}\left(\mathbf{x}_{+}^{-}\right)\right\|_{q, t(x)}^{s}(r) \mathrm{d} x=\int_{\mathbb{X}^{t}} \mathrm{~d} x \int_{\mathcal{X}^{t(x)}}\left\|\mathrm{M}_{+}^{-}(x \sqcup v)\right\|_{q, t(x)}^{s}(r) \mathrm{d} v \\
& =\int_{\mathcal{X}^{t}}\left\|\mathrm{M}_{+}^{-}(v)\right\|_{q, t}^{s}(r) \mathrm{d} v-\left\|\mathrm{M}_{+}^{-}(\emptyset)\right\|_{q, t}^{s}(r)=\|\mathrm{M}\|_{q, t}^{s}(r)-\left\|\mathrm{M}_{+}^{-}(\emptyset)\right\|_{q, t}^{s}(r) .
\end{aligned}
$$

For the estimate of $\mathrm{D}_{+}^{\circ}$ we shall require the use of the norm

where

$$
\left\|\mathrm{M}_{+}^{\circ}\right\|_{q, t}^{s}(r):=\left(\int_{\mathcal{X}^{t}}\left(\left\|\mathrm{M}_{+}^{\circ}\left(v_{+}^{\circ}\right)\right\|_{q, t}^{s}(r)\right)^{2} r\left(v_{+}^{\circ}\right) \mathrm{d} v_{+}^{\circ}\right)^{1 / 2}
$$

$$
\left\|\mathrm{M}_{+}^{\circ}\left(v_{+}^{\circ}\right)\right\|_{q, t}^{s}(r):=\left(\int_{\mathcal{X}^{t}}\left(\int_{\mathcal{X}^{t}} \underset{v_{\circ}^{\circ} \in \mathcal{X}^{t}}{\operatorname{ess} \sup } s\left(v_{\circ}^{\circ}\right)\|\mathrm{M}(\boldsymbol{v})\|_{q} \mathrm{~d} v_{+}^{-}\right)^{2} r\left(v_{\circ}^{-}\right) \mathrm{d} v_{\circ}^{-}\right)^{1 / 2}
$$

in particular, $\left\|\mathrm{M}_{+}^{\circ}\right\|_{q, t}^{s}(r) \leq\|\mathrm{M}\|_{q, t}^{s}(r)$. So we have

$$
\begin{aligned}
\left\|\mathrm{D}_{+}^{\circ}\right\|_{p, t}^{(2)}(r)^{2} & \leq \int_{\mathbb{X}^{t}}\left(\left\|\dot{\mathrm{M}}_{+}^{\circ}\left(\mathbf{x}_{+}^{\circ}\right)\right\|_{q, t(x)}^{s}(r)\right)^{2} r(x) \mathrm{d} x \\
& =\left\|\mathrm{M}_{+}^{\circ}\right\|_{q, t}^{s}(r)^{2}-\left\|\mathrm{M}_{+}^{\circ}(\emptyset)\right\|_{q, t}^{s}(r)^{2} \leq\|\mathrm{M}\|_{q, t}^{s}(r)^{2} .
\end{aligned}
$$

In a similar manner we obtain the estimate for $\mathrm{D}_{\circ}^{-}$,

$$
\begin{aligned}
\left\|\mathrm{D}_{\circ}^{-}\right\|_{p, t}^{(2)}(r)^{2} & \leq \int_{\mathbb{X}^{t}}\left(\left\|\dot{\mathrm{M}}_{\circ}^{-}\left(\mathbf{x}_{\circ}^{-}\right)\right\|_{q, t(x)}^{s}(r)\right)^{2} r(x) \mathrm{d} x \\
& =\left\|\mathrm{M}_{\circ}^{-}\right\|_{q, t}^{s}(r)^{2}-\left\|\mathrm{M}_{\circ}^{-}(\emptyset)\right\|_{q, t}^{s}(r)^{2} \leq\|\mathrm{M}\|_{q, t}^{s}(r)^{2} .
\end{aligned}
$$

Finally, from $\left\|\mathrm{D}_{\circ}^{\circ}(x)\right\|_{p} \leq\left\|\dot{\mathrm{M}}\left(\mathbf{x}_{\circ}^{\circ}\right)\right\|_{q, t(x)}^{s}(r)$ we similarly obtain

$$
\left\|\mathrm{D}_{\circ}^{\circ}\right\|_{p, t}^{(\infty)}(s) \leq \underset{x \in \mathbb{X}^{t}}{\operatorname{ess} \sup }\left\{s(x)\left\|\dot{\mathrm{M}}\left(\mathbf{x}_{\circ}^{\circ}\right)\right\|_{q, t(x)}^{s}(r)\right\} \leq\|\mathrm{M}\|_{q, t}^{s}(r)
$$

if $p \geq r^{-1}+q+s^{-1}$, which concludes the proof.

Since $\mathrm{T}_{t}=\boldsymbol{\imath}_{0}^{t}(\mathrm{M})$ the theorem obviously states that $\left\|\boldsymbol{\imath}_{0}^{t}(\mathrm{M})\right\|_{p} \leq\|\mathrm{M}\|_{q, t}^{s}(r)$ for all $p \geq \frac{1}{r}+q+\frac{1}{s}$, and in particular for the case when $\mathrm{M}(\boldsymbol{v})=0$ if $\sum\left|v_{\nu}^{\mu}\right| \neq 1$, and $\mathrm{M}(\mathbf{x})=\mathrm{D}(\mathbf{x})$. Then the result of the theorem becomes

$$
\left\|\boldsymbol{i}_{0}^{t}(\mathbf{D})\right\|_{p} \leq\|\mathrm{D}\|_{q, t}^{s}(r)
$$


and now we shall begin to evaluate the quantum stochastic norm explicitly for the single integrand $\mathrm{D}(\mathbf{x})$,

$$
\|\mathrm{D}\|_{q, t}^{s}(r)=\left\|\mathrm{D}_{+}^{-}\right\|_{q, t}^{(1)}+\left\|\mathrm{D}_{+}^{-}(\emptyset)\right\|_{q, t}^{s}(r) .
$$

One may now proceed in a similar manner to find that

$$
\left\|\mathrm{D}_{+}^{-}(\emptyset)\right\|_{q, t}^{s}(r)=\left\|\mathrm{D}_{\circ}^{-}\right\|_{q, t}^{(2)}(r)+\left\|\mathrm{D}_{+}^{\circ}\right\|_{q, t}^{(2)}(r)+\left\|\mathrm{D}_{\circ}^{\circ}\right\|_{q, t}^{(\infty)}(s),
$$

and thus we have recovered the inequality stated at the beginning of this section.

6. Adapted and Q-adapted QS integrals. The quantum-stochastic integral (7) constructed in [7, as well as its single variations [2] introduced in [6], are defined explicitly and do not require that the functions $\mathrm{M}$ and $\mathbf{D}$ under the integral be adapted. By virtue of the continuity we have proved above, they can be approximated in the inductive convergence by the sequence of integral sums $\boldsymbol{i}_{0}^{t}\left(\mathrm{M}_{n}\right), \boldsymbol{i}_{0}^{t}\left(\mathbf{D}_{n}\right)$ corresponding to step measurable operator-functions $\mathbf{M}_{n}$ and $\mathbf{D}_{n}$ if the latter converge inductively to $\mathbf{M}$ and $\mathbf{D}$ in the poly-norm (8).

In fact, if there exist functions $r, s$ with $r^{-1}, s^{-1} \in \mathfrak{p}_{0}$ and $q \in \mathfrak{p}_{1}$ such that $\left\|\mathrm{M}_{n}-\mathrm{M}\right\|_{q, t}^{s}(r) \rightarrow 0$, then there also exists a function $p \in \mathfrak{p}_{1}$ such that $\left\|\boldsymbol{\imath}_{0}^{t}\left(\mathrm{M}_{n}-\mathrm{M}\right)\right\|_{p} \rightarrow 0$, and we have $p \geq r^{-1}+q+s^{-1}$ by the inequality 10 , which implies the inductive convergence $\boldsymbol{\imath}_{0}^{t}\left(\mathrm{M}_{n}\right) \rightarrow \boldsymbol{\imath}_{0}^{t}(\mathrm{M})$ as a result of the linearity of $\boldsymbol{\imath}_{0}^{t}$.

Let $\mathrm{Q}: x \mapsto \mathfrak{L}\left(\mathfrak{k}_{x}\right)$ be a measurable operator-valued function with $q$-contractive values $\|\mathrm{Q}(x)\| \leq q(x)$ with respect to a positive function $q \in \mathfrak{p}$, where $\mathfrak{L}\left(\mathfrak{k}_{x}\right)$ is the space of adjointable maps in $\mathfrak{k}_{x}$. We shall say that the integrand $\mathbf{D}(x)$ is Q-adapted if it has the product form

$$
\mathrm{D}_{\nu}^{\mu}(x)=\mathrm{D}_{\nu}^{\mu}(x)^{t(x)} \otimes \mathrm{Q}_{t(x)}^{\otimes}
$$

with respect to the Fock split $\mathcal{G}=\mathcal{G}^{t(x)} \otimes \mathcal{F}_{[t(x)}$ corresponding to $\mathbb{X}=\mathbb{X}^{t(x)} \sqcup \mathbb{X}_{[t(x)}$, where $\mathrm{D}_{\nu}^{\mu}(x)^{t(x)}:=\mathrm{K}_{\nu}^{\mu}(x)$ is the restricted action $G_{+}^{t(x)} \rightarrow G_{-}^{t(x)}$ of $\mathrm{D}_{\nu}^{\mu}(x)$ corresponding to the vacuum embeddings $G_{+}^{t(x)} \subseteq G_{+}$and $G_{-}^{t(x)} \subseteq G_{-}$. We can now write the Q-adapted QS integrals as a more explicit form of the general QS integrals (3)

$$
\begin{aligned}
& {\left[\Lambda_{-}^{+}\left(\mathrm{D}_{+}^{-}, \triangle\right) \chi\right](\vartheta)=\int_{\triangle}\left[\mathrm{K}_{+}^{-}(x) \otimes \mathrm{Q}^{\otimes}(\varkappa) \dot{\chi}(\varkappa)\right]\left(\vartheta^{t(x)}\right) \mathrm{d} x, \quad x \notin \vartheta,} \\
& {\left[\Lambda_{\circ}^{+}\left(\mathrm{D}_{+}^{\circ}, \triangle\right) \chi\right](\vartheta)=\sum_{x \in \triangle \cap \vartheta}\left[\mathrm{K}_{+}^{\circ}(x) \otimes \mathrm{Q}^{\otimes}(\varkappa) \stackrel{\chi}{\chi}(\varkappa)\right]\left(\vartheta^{t(x)}\right),} \\
& {\left[\Lambda_{-}^{\circ}\left(\mathrm{D}_{\circ}^{-}, \triangle\right) \chi\right](\vartheta)=\int_{\triangle}\left[\mathrm{K}_{\circ}^{-}(x) \otimes \mathrm{Q}^{\otimes}(\varkappa) \stackrel{\circ}{\chi}(x \sqcup \varkappa)\right]\left(\vartheta^{t(x)}\right) \mathrm{d} x, \quad x \notin \vartheta,} \\
& {\left[\Lambda_{\circ}^{\circ}\left(\mathrm{D}_{\circ}^{\circ}, \triangle\right) \chi\right](\vartheta)=\sum_{x \in \triangle \cap \vartheta}\left[\mathrm{K}_{\circ}^{\circ}(x) \otimes \mathrm{Q}^{\otimes}(\varkappa) \dot{\chi}(x \sqcup \varkappa)\right]\left(\vartheta^{t(x)}\right) .}
\end{aligned}
$$

Here $\mathrm{Q}^{\otimes}(\varkappa)=\bigotimes_{x \in \varkappa} \mathrm{Q}(x)$ for any $\varkappa \in \mathcal{X}_{t(x)}$ and $\dot{\chi}\left(\varkappa, \vartheta^{t}\right)=\chi\left(\vartheta^{t} \sqcup \varkappa\right)$ on the decomposition of the chain $\vartheta \in \mathcal{X}$ into $\vartheta^{t}=\{x \in \vartheta: t(x)<t\}$ and $\varkappa=\{x \in \vartheta: t(x)>t\} \equiv \vartheta_{t}$. The usual adapted case corresponds to the identity operator $\mathrm{Q}=\mathrm{I}$ which we shall now refer to as Bosonic adapted, however there are also two other basic cases of interest. The first is the vacuum adapted case corresponding to the zero operator $\mathrm{Q}=\mathrm{O}$, and the second is the Fermionic adapted case corresponding to $\mathrm{Q}=-\mathrm{I}$. In the vacuum adapted case 
$\mathrm{Q}^{\otimes}=\mathrm{O}^{\otimes}$ is the vacuum projector given on $\mathcal{G}_{*}$ as

$$
\left[\mathrm{P}_{0} \chi\right](\vartheta)=\chi(\emptyset) \delta_{\emptyset}(\vartheta), \quad \delta_{\emptyset}(\vartheta)= \begin{cases}1 & \vartheta=\emptyset \\ 0 & \vartheta \neq \emptyset .\end{cases}
$$

The \pm I-adapted integrands define the Fermionic and Bosonic fields in the GuichardetFock space as

$$
\Lambda\left(\mathbf{D}_{\circ}^{-}\right)=\Lambda_{-}^{\circ}\left(\mathrm{D}_{\circ}^{-}\right), \quad \Lambda\left(\mathbf{D}_{+}^{\circ}\right)=\Lambda_{\circ}^{+}\left(\mathrm{D}_{+}^{\circ}\right),
$$

with $\mathrm{D}_{\circ}^{-}(x)=\mathrm{K}_{\circ}^{-}(x) \otimes \mathrm{Q}_{t(x)}^{\otimes}$, and $\mathrm{D}_{+}^{\circ}(x)=\mathrm{K}_{+}^{\circ}(x) \otimes \mathrm{Q}_{t(x)}^{\otimes}$, and the terminology used here is justified in the corollary following the next proposition.

Proposition 6.1. Let $\Lambda\left(\mathbf{D}_{\circ}^{-}\right)$and $\Lambda\left(\mathbf{D}_{+}^{\circ}\right)$ be $\mathrm{Q}$-adapted quantum stochastic integrals with respect to a q-contractive operator $\mathrm{Q}$ on $\mathcal{K}_{*}$. Suppose that both $\mathrm{K}_{\circ}^{-}(x)$ and $\mathrm{K}_{+}^{\circ}(z)$ are diagonal on $\mathcal{X}^{t(x)}$ such that $\left[\mathrm{K}_{+}^{\circ}(x) \chi\right](\vartheta)=\mathrm{K}_{+}^{\circ}(x, \vartheta) \chi(\vartheta)$ and $\left[\mathrm{K}_{\circ}^{-}(x) \dot{\chi}(x)\right](\vartheta)=$ $\mathrm{K}_{\circ}^{-}(x, \vartheta) \chi(x \sqcup \vartheta)$, and that the commutation $\left[\mathrm{K}_{\circ}^{-}(x), \mathrm{K}_{+}^{\circ}(z)\right]=0$ is satisfied for each $x, z \in \mathbb{X}^{t}$ with $x \neq z$, then given constant $\mathrm{Q}=c \mathrm{I}, c \in \mathbb{C}$, the c-commutator

$$
\left[\Lambda\left(\mathbf{D}_{\circ}^{-}\right), \Lambda\left(\mathbf{D}_{+}^{\circ}\right)\right]_{c}=\Lambda\left(\mathbf{D}_{\circ}^{-}\right) \Lambda\left(\mathbf{D}_{+}^{\circ}\right)-c \Lambda\left(\mathbf{D}_{+}^{\circ}\right) \Lambda\left(\mathbf{D}_{\circ}^{-}\right)
$$

satisfies the equation

$$
\left[\Lambda\left(\mathbf{D}_{\circ}^{-}, t\right), \Lambda\left(\mathbf{D}_{+}^{\circ}, t\right)\right]_{c}=\int_{\mathbb{X}^{t}} \mathrm{~K}_{\circ}^{-}(x) \mathrm{K}_{+}^{\circ}(x) \mathrm{d} x \otimes\left(\mathrm{Q}^{2}\right)_{t}^{\otimes}
$$

if $\mathrm{D}_{\circ}^{-}\left(x, \vartheta^{t(x)} \backslash z\right)=\mathrm{D}_{\circ}^{-}\left(x, \vartheta^{t(x)}\right)$ for all $x>z$, and $\mathrm{D}_{+}^{\circ}\left(z, \vartheta^{t(z)} \sqcup x\right)=\mathrm{D}_{+}^{\circ}\left(z, \vartheta^{t(z)}\right)$ for all $z>x$.

Proof. The QS integrals are defined as

$$
\left[\Lambda\left(\mathbf{D}_{\circ}^{-}, t\right) \chi\right](\vartheta)=\int_{\mathbb{X}^{t}} \mathrm{D}_{\circ}^{-}\left(x, \vartheta^{t(x)}\right) \otimes \mathrm{Q}^{\otimes\left|\vartheta_{t(x)}\right|} \chi(\vartheta \sqcup x) \mathrm{d} x
$$

and

$$
\left[\Lambda\left(\mathbf{D}_{+}^{\circ}, t\right) \chi\right](\vartheta)=\sum_{z \in \vartheta^{t}} \mathrm{D}_{+}^{\circ}\left(z, \vartheta^{t(z)}\right) \otimes \mathrm{Q}^{\otimes\left|\vartheta_{t(z)}\right|} \chi(\vartheta \backslash z)
$$

Consider the quantity

$$
\begin{aligned}
& {\left[\Lambda\left(\mathbf{D}_{\circ}^{-}\right) \Lambda\left(\mathbf{D}_{+}^{\circ}\right) \chi\right](\vartheta)-\left[\int_{\mathbb{X}^{t}} \mathrm{~K}_{\circ}^{-}(x) \mathrm{K}_{+}^{\circ}(x) \mathrm{d} x \otimes\left(\mathrm{Q}_{t}^{2}\right)^{\otimes} \chi\right](\vartheta)} \\
& =\int_{\mathbb{X}^{t}} \mathrm{D}_{\circ}^{-}\left(x, \vartheta^{t(x)}\right) \otimes \mathrm{Q}^{\otimes\left|\vartheta_{t(x)}\right|} \sum_{z \in \vartheta^{t(x)}} \mathrm{D}_{+}^{\circ}\left(z, \vartheta^{t(z)}\right) \otimes \mathrm{Q}^{\otimes\left|\vartheta_{t(z)} \sqcup x\right|} \chi(x \sqcup \vartheta \backslash z) \mathrm{d} x \\
& \quad+\int_{\mathbb{X}^{t}} \mathrm{D}_{\circ}^{-}\left(x, \vartheta^{t(x)}\right) \otimes \mathrm{Q}^{\otimes\left|\vartheta_{t(x)}\right|} \sum_{z \in \vartheta_{t(x)}^{t}} \mathrm{D}_{+}^{\circ}\left(z, \vartheta^{t(z)} \sqcup x\right) \otimes \mathrm{Q}^{\otimes\left|\vartheta_{t(z)}\right|} \chi(x \sqcup \vartheta \backslash z) \mathrm{d} x,
\end{aligned}
$$

where $\vartheta_{s}^{t}=\vartheta \cap(s, t)$. Since $\mathrm{Q}=c \mathrm{I}$, and by the requirement that $\mathrm{D}_{+}^{\circ}\left(z, \vartheta^{t(z)} \sqcup x\right)=$ $\mathrm{D}_{+}^{\circ}\left(z, \vartheta^{t(z)}\right)$ for all $z>x$, we can write this as

$$
\begin{aligned}
= & \sum_{z \in \vartheta^{t}} \int_{\mathbb{X}_{t(z)}^{t}} \mathrm{D}_{\circ}^{-}\left(x, \vartheta^{t(x)}\right) \otimes \mathrm{Q}^{\otimes\left|\vartheta_{t(x)}\right|} \mathrm{D}_{+}^{\circ}\left(z, \vartheta^{t(z)}\right) \otimes c \mathrm{Q}^{\otimes\left|\vartheta_{t(z)}\right|} \chi(x \sqcup \vartheta \backslash z) \mathrm{d} x \\
& +\sum_{z \in \vartheta^{t}} \int_{\mathbb{X}^{t(z)}} \mathrm{D}_{\circ}^{-}\left(x, \vartheta^{t(x)}\right) \otimes \mathrm{Q}^{\otimes\left|\vartheta_{t(x)}\right|} \mathrm{D}_{+}^{\circ}\left(z, \vartheta^{t(z)}\right) \otimes \mathrm{Q}^{\otimes\left|\vartheta_{t(z)}\right|} \chi(x \sqcup \vartheta \backslash z) \mathrm{d} x,
\end{aligned}
$$


and now we look at

$$
\begin{aligned}
& c\left[\Lambda\left(\mathbf{D}_{+}^{\circ}, t\right) \Lambda\left(\mathbf{D}_{\circ}^{-}, t\right) \chi\right](\vartheta) \\
& =c \sum_{z \in \vartheta^{t}} \mathrm{D}_{+}^{\circ}\left(z, \vartheta^{t(z)}\right) \otimes \mathrm{Q}^{\otimes\left|\vartheta_{t(z)}\right|} \int_{\mathbb{X}^{t(z)}} \mathrm{D}_{\circ}^{-}\left(x, \vartheta^{t(x)}\right) \otimes \mathrm{Q}^{\otimes\left|\vartheta_{t(x)} \backslash z\right|} \chi(x \sqcup \vartheta \backslash z) \mathrm{d} x \\
& \quad+c \sum_{z \in \vartheta^{t}} \mathrm{D}_{+}^{\circ}\left(z, \vartheta^{t(z)}\right) \otimes \mathrm{Q}^{\otimes\left|\vartheta_{t(z)}\right|} \int_{\mathbb{X}_{t(z)}^{t}} \mathrm{D}_{\circ}^{-}\left(x, \vartheta^{t(x)} \backslash z\right) \otimes \mathrm{Q}^{\otimes\left|\vartheta_{t(x)}\right|} \chi(x \sqcup \vartheta \backslash z) \mathrm{d} x
\end{aligned}
$$

which we can write as

$$
\begin{aligned}
= & \sum_{z \in \vartheta^{t}} \mathrm{D}_{+}^{\circ}\left(z, \vartheta^{t(z)}\right) \otimes \mathrm{Q}^{\otimes\left|\vartheta_{t(z)}\right|} \int_{\mathbb{X}^{t(z)}} \mathrm{D}_{\circ}^{-}\left(x, \vartheta^{t(x)}\right) \otimes \mathrm{Q}^{\otimes\left|\vartheta_{t(x)}\right|} \chi(x \sqcup \vartheta \backslash z) \mathrm{d} x \\
& +c \sum_{z \in \vartheta^{t}} \mathrm{D}_{+}^{\circ}\left(z, \vartheta^{t(z)}\right) \otimes \mathrm{Q}^{\otimes\left|\vartheta_{t(z)}\right|} \int_{\mathbb{X}_{t(z)}^{t}} \mathrm{D}_{\circ}^{-}\left(x, \vartheta^{t(x)}\right) \otimes \mathrm{Q}^{\otimes\left|\vartheta_{t(x)}\right|} \chi(x \sqcup \vartheta \backslash z) \mathrm{d} x,
\end{aligned}
$$

and indeed the result follows as a consequence of the commutation $\left[\mathrm{K}_{\circ}^{-}(x), \mathrm{K}_{+}^{\circ}(z)\right]=0$ for each $x, z \in \mathbb{X}^{t}$ with $x \neq z$.

COROLlary 6.2. In particular notice that the Bosonic and Fermionic field commutators are obtained, respectively, in the cases where $c=+1$ and $c=-1$, where we have

$$
\left[\Lambda\left(\mathbf{D}_{\circ}^{-}, t\right), \Lambda\left(\mathbf{D}_{+}^{\circ}, t\right)\right]_{ \pm 1}=\int_{\mathbb{X}^{t}} \mathrm{~K}_{\circ}^{-}(x) \mathrm{K}_{+}^{\circ}(x) \mathrm{d} x \otimes \mathrm{I}_{t}^{\otimes}
$$

and the monotonic field commutator, $c=0$, is

$$
\left[\Lambda\left(\mathbf{D}_{\circ}^{-}, t\right), \Lambda\left(\mathbf{D}_{+}^{\circ}, t\right)\right]_{0}=\int_{\mathbb{X}^{t}} \mathrm{~K}_{\circ}^{-}(x) \mathrm{K}_{+}^{\circ}(x) \mathrm{d} x \otimes \mathrm{O}_{t}^{\otimes}
$$

where we have made use of the identification $\mathrm{D}_{\nu}^{\mu}(\emptyset)=0$.

Obviously the QS integral $\mathrm{Y}(t)=\boldsymbol{i}_{0}^{t}(\mathbf{D})$ of any Q-adapted integrand $\mathbf{D}(x)$ is an operator-valued Q-adapted process in the sense that $\mathrm{Y}(t)=\mathrm{Y}^{t} \otimes \mathrm{Q}_{t}^{\otimes}$. The approximation of this integral in the class of adapted step functions when $\mathrm{Q}=\mathrm{I}$, by continuity, leads to the usual definition of the quantum-stochastic integral $\boldsymbol{i}_{0}^{t}(\mathbf{D})$ which was given by Hudson and Parthasarathy for the identity-adapted case with $\mathbb{X}=\mathbb{R}_{+}, t(x)=x$ as the weak limit of integral sums

$$
\boldsymbol{i}_{0}^{t}\left(\mathbf{D}_{n}\right)=\int_{0}^{t} \Lambda\left(\mathbf{D}_{n}, \mathrm{~d} x\right)=\sum_{j=1}^{n} \mathrm{D}_{\nu}^{\mu}\left(x_{j}\right) \mathrm{A}_{\mu}^{\nu}\left(\triangle_{j}\right) .
$$

Here $\mathbf{D}\left(x_{j}\right)=\mathbf{D}_{n}(x)$ for $x \in\left[x_{j}, x_{j+1}\right)$ is an adapted approximation corresponding to the decomposition $\mathbb{R}_{+}=\sum_{j=1}^{n} \triangle_{i}$ into the intervals $\triangle_{j}=\left[x_{j}, x_{j+1}\right)$ given by the chain $x_{0}=0<x_{1}<\ldots<x_{n}<x_{n+1}=\infty$, and $\mathrm{D}_{\nu}^{\mu}(x) \mathrm{A}_{\mu}^{\nu}(\triangle)$ is the sum of the operators (3) with functions $\mathrm{D}_{\nu}^{\mu}(x)$ constant on $\triangle$ which can therefore be pulled out in front of the integrals $\mathrm{A}_{\mu}^{\nu}$.

In particular, for $\mathrm{D}_{+}^{-}=0=\mathrm{D}_{\circ}^{\circ}$ and $\mathrm{D}_{\circ}^{-}=g \otimes \hat{1}=\mathrm{D}_{+}^{\circ}$, where $\hat{1}=\mathrm{I}^{\otimes}$ is the unit operator in $\mathcal{F}_{*}$ and $g(x)$ is a scalar locally square integrable function corresponding to the case $\mathfrak{k}_{x}=\mathbb{C}=\mathfrak{h}$, we obtain the Itô definition of the Wiener integral

$$
\dot{I}_{0}^{t}(g)=\int_{0}^{t} g(x) w(\mathrm{~d} x), \quad \int_{0}^{t} g(x) \widehat{w}(\mathrm{~d} x)=i_{0}^{t}(\mathbf{D})
$$


with respect to the stochastic measure $w(\triangle), \triangle \in \mathfrak{F}_{\mathbb{X}}$ on $\mathbb{R}_{+}$, represented in $\mathcal{G}_{*}=\mathcal{F}_{*}$ by the operators $\widehat{w}(\triangle)=\mathrm{A}_{\circ}^{+}(\triangle)+\mathrm{A}_{-}^{\circ}(\triangle)$. We also note that the multiple integral (7) in the trivially adapted case $\mathrm{M}(\boldsymbol{v})=M(\boldsymbol{v}) \otimes \mathrm{I}^{\otimes}$ defines the Fock representation of the generalized Maassen-Meyer kernels [9, 17] and in the case

$$
M(\boldsymbol{v})=m\left(v_{\circ}^{-} \sqcup v_{+}^{\circ}\right) \delta_{\emptyset}\left(v_{+}^{-}\right) \delta_{\emptyset}\left(v_{\circ}^{\circ}\right), \quad \delta_{\emptyset}(v)= \begin{cases}1, & v=\emptyset, \\ 0, & v \neq \emptyset,\end{cases}
$$

it leads to the multiple stochastic integrals $\boldsymbol{\imath}_{0}^{t}(\mathrm{M})=\widehat{I}_{0}^{t}(m)$,

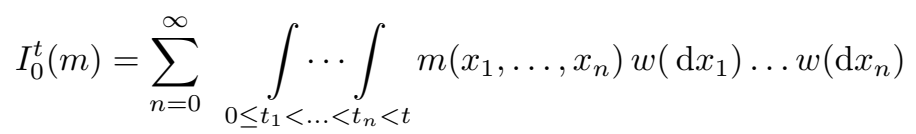

of the generalized functions $m \in \bigcup_{r^{-1} \in \mathfrak{p}_{0}} G_{\star}(r)$, that is, to the Hida distributions [11, 13] of the Wiener measure $w(\triangle)$ represented as $\widehat{w}(\triangle)$. Thus, we can consider the trivially adapted QS multiple integrals $\boldsymbol{\imath}_{0}^{t}(\mathrm{M})$ as quantum Hida operator-distributions whose properties are described in the following corollary when $\mathrm{Q}=\mathrm{I}$.

COROLlary 6.3. Suppose that $\mathrm{M}(\boldsymbol{v})=M(\boldsymbol{v}) \otimes \mathrm{Q}^{\otimes}$ where $\left\|\mathrm{Q}^{\otimes}\right\|_{q}=1$, i.e. the operatorfunction $\mathrm{M}$ is defined by the q-contractive ampliation of the $\star$-kernel $M$ with $\|M\|_{t}^{s}(r)<\infty$,

$$
M\left(\begin{array}{cc}
v_{+}^{-} & v_{\circ}^{-} \\
v_{+}^{\circ} & v_{\circ}^{\circ}
\end{array}\right): \mathfrak{k}^{\otimes}\left(v_{\circ}^{-} \sqcup v_{\circ}^{\circ}\right) \otimes \mathfrak{h} \rightarrow \mathfrak{k}^{\otimes}\left(v_{\circ}^{\circ} \sqcup v_{+}^{\circ}\right) \otimes \mathfrak{h},
$$

where

$$
\|M\|_{t}^{s}(r)=\int_{\mathcal{X}^{t}} \mathrm{~d} v_{+}^{-}\left(\int_{\mathcal{X}^{t}} \mathrm{~d} v_{+}^{\circ} \int_{\mathcal{X}^{t}} \mathrm{~d} v_{\circ}^{-} \underset{v_{\circ}^{\circ} \in \mathcal{X}^{t}}{\operatorname{ess} \sup }\left\{s\left(v_{\circ}^{\circ}\right)\|M(\boldsymbol{v})\|\right\}^{2} r\left(v_{+}^{\circ} \sqcup v_{\circ}^{-}\right)\right)^{1 / 2}
$$

for all $t \in \mathbb{R}_{+}$and for some $r(v)=\prod_{x \in v} r(x), s(v)=\prod_{x \in v} s(x) ; r^{-1}, s^{-1} \in \mathfrak{p}_{0}$. Then the integral (7) defines a $\mathrm{Q}$-adapted family $\mathrm{T}_{t}, t \in \mathbb{R}_{+}$, of $p$-bounded operators $\mathrm{T}_{t}=\boldsymbol{\imath}_{0}^{t}\left(M \otimes \mathrm{Q}^{\otimes}\right),\left\|\mathrm{T}_{t}\right\|_{p} \leq\|M\|_{t}^{s}(r)$ for $p \geq r^{-1}+q+s^{-1}$, with bounded Q-adapted quantum-stochastic derivatives $\mathrm{D}_{\nu}^{\mu}(x)=\boldsymbol{\imath}_{0}^{t(x)}\left(\dot{M}\left(\mathrm{x}_{\nu}^{\mu}\right) \otimes \mathrm{Q}^{\otimes}\right)$.

Proof. Since $\left\|M(\boldsymbol{v}) \otimes \mathrm{Q}^{\otimes}\right\|_{q} \leq\|M(\boldsymbol{v})\|$ the result immediately follows from the inequality $\left\|M \otimes \mathrm{Q}^{\otimes}\right\|_{q, t}^{s}(r) \leq\|M\|_{t}^{s}(r)$.

\section{References}

[1] L. Accardi, F. Fagnola, Stochastic integration, in: Quantum Probability and Applications III (Oberwolfach, 1987), Lecture Notes in Math. 1303, Springer, Berlin, 1988, 6-19.

[2] L. Accardi, J. Quaegebeur, The Itô algebra of quantum Gaussian fields, J. Funct. Anal. 85 (1989), 213-263.

[3] V. P. Belavkin, A reconstruction theorem for quantum stochastic processes, Teoret. Mat. Fiz. 62 (1985), 408-431; English transl.: Theoret. and Math. Phys. 62 (1985), 275-289.

[4] V. P. Belavkin, Optimal non-linear filtering of quantum signals, in: Proceedings of 9th Conference on Coding Theory and Information Transmission, University of Odessa, 1988, $342-345$. 
[5] V. P. Belavkin, Nondemolition measurements, nonlinear filtering, and dynamic programming of quantum stochastic processes, in: Modeling and Control of Systems in Engineering, Quantum Mechanics, Economics and Biosciences (Sophia-Antipolis, 1988), Lecture Notes in Control and Inform. Sci. 121, Springer, Berlin, 1989, 245-265.

[6] V. P. Belavkin, A quantum stochastic calculus in Fock space of input and output nondemolition processes, in: Quantum Probability and Applications V (Heidelberg, 1988), Lecture Notes in Math. 1442, Springer, Berlin, 1990, 99-125.

[7] V. P. Belavkin, A quantum nonadapted Itô formula and stochastic analysis in Fock scale, J. Funct. Anal. 102 (1991), 414-447.

[8] V. P. Belavkin, Quantum stochastic calculus and quantum non-linear filtering, J. Multivariate Anal. 42 (1992), 171-201.

[9] M. P. Evans, R. L. Hudson, Multidimensional quantum diffusions, in: Quantum Probability and Applications III (Oberwolfach, 1987), Lecture Notes in Math. 1303, Springer, Berlin, 1988, 69-88.

[10] A. Guichardet, Symmetric Hilbert Spaces and Related Topics, Lecture Notes in Math. 261, Springer, Berlin, 1972.

[11] T. Hida, Brownian Motion, Applications of Mathematics 11, Springer, New York, 1980.

[12] A. S. Holevo, Time-ordered exponentials in quantum stochastic calculus, Preprint 517, Universität Heidelberg, 1989.

[13] Yu. G. Kondratiev, P. Leukert, J. Potthoff, L. Streit, W. Westerkamp, Generalized functionals in Gaussian spaces: the characterization theorem revisited, J. Funct. Anal. 141 (1996), 301-318.

[14] J. M. Lindsay, On set convolutions and integral-sum kernel operators, in: Probability Theory and Mathematical Statistics (Vilnius, 1989), vol. II, Mokslas, Vilnius, 1990, 105-123.

[15] J. M. Lindsay, H. Maassen, The Stochastic Calculus of Bose Noise, CWI Syllabus 32, Centre for Mathematics and Computer Science, Amsterdam, 1992.

[16] P. Malliavin, Stochastic calculus of variations and hypoelliptic operators, in: Proceedings of the International Symposium on Stochastic Differential Equations (Res. Inst. Math. Sci., Kyoto, 1976), Wiley, New York, 1978, 195-263.

[17] P. A. Meyer, Éléments de probabilités quantiques, in: Séminaire de Probabilités, XXI, Lecture Notes in Math. 1247, Springer, Berlin, 1987, 33-78.

[18] K. R. Parthasarathy, K. B. Sinha, Stochastic integral representation of bounded quantum martingales in Fock space, J. Funct. Anal. 67 (1986), 126-151.

[19] A. V. Skorokhod, On a generalization of the stochastic integral, Teor. Veroyatn. Primen. 20 (1975), 223-228; English transl.: Theory Probab. Appl. 20 (1975), 219-233. 